\title{
Scalable Private Set Intersection Based on OT Extension
}

\author{
BENNY PINKAS, Bar-Ilan University \\ THOMAS SCHNEIDER and MICHAEL ZOHNER, Technische Universität Darmstadt
}

Private set intersection (PSI) allows two parties to compute the intersection of their sets without revealing any information about items that are not in the intersection. It is one of the best studied applications of secure computation and many PSI protocols have been proposed. However, the variety of existing PSI protocols makes it difficult to identify the solution that performs best in a respective scenario, especially since they were not compared in the same setting. In addition, existing PSI protocols are several orders of magnitude slower than an insecure naïve hashing solution, which is used in practice.

In this article, we review the progress made on PSI protocols and give an overview of existing protocols in various security models. We then focus on PSI protocols that are secure against semi-honest adversaries and take advantage of the most recent efficiency improvements in Oblivious Transfer (OT) extension, propose significant optimizations to previous PSI protocols, and suggest a new PSI protocol whose runtime is superior to that of existing protocols. We compare the performance of the protocols, both theoretically and experimentally, by implementing all protocols on the same platform, give recommendations on which protocol to use in a particular setting, and evaluate the progress on PSI protocols by comparing them to the currently employed insecure naïve hashing protocol. We demonstrate the feasibility of our new PSI protocol by processing two sets with a billion elements each.

\section{CCS Concepts: • Security and privacy $\longrightarrow$ Privacy-preserving protocols;}

Additional Key Words and Phrases: Privacy-preserving protocols, Pseudonymity, Anonymity and untraceability

\section{ACM Reference format:}

Benny Pinkas, Thomas Schneider, and Michael Zohner. 2018. Scalable Private Set Intersection Based on OT Extension. ACM Trans. Priv. Secur. 21, 2, Article 7 (January 2018), 35 pages.

https://doi.org/10.1145/3154794

This article is a combined and extended version of Pinkas et al. (2014) (USENIX'14) and Pinkas et al. (2015) (USENIX 2015) with substantial improvements summarized in Section 1.4.

This article was supported by the European Union's 7th Framework Program (FP7/2007-2013) under Grant Agreement No. 609611 (PRACTICE), by the DFG as part of Project E4 within the CRC 1119 CROSSING, by the German Federal Ministry of Education and Research (BMBF) within EC SPRIDE and CRISP, by the Hessian LOEWE excellence initiative within CASED, by a grant from the Israel Ministry of Science and Technology (Grant No. 3-9094), by the BIU Center for Research in Applied Cryptography and Cyber Security in conjunction with the Israel National Cyber Bureau in the Prime Minister's Office, and by the National Science Foundation, under Grant No. CNS-0435060, Grant No. CCR-0325197 and Grant EN-CS-0329609. We thank Oleksandr Tkachenko for the implementation of the PSI protocol for billion-element sets.

Authors' addresses: B. Pinkas, Center for Research in Applied Cryptography and Cyber Security, Bar-Ilan University, 5290002, Ramat-Gan, Israel; T. Schneider and M. Zohner, Center for Research in Security and Privacy, TU Darmstadt, Mornewegstraße 30, 64289 Darmstadt, Germany.

Permission to make digital or hard copies of all or part of this work for personal or classroom use is granted without fee provided that copies are not made or distributed for profit or commercial advantage and that copies bear this notice and the full citation on the first page. Copyrights for components of this work owned by others than the author(s) must be honored. Abstracting with credit is permitted. To copy otherwise, or republish, to post on servers or to redistribute to lists, requires prior specific permission and/or a fee. Request permissions from permissions@acm.org.

(c) 2018 ACM 2471-2566/2018/01-ART7 $\$ 15.00$

https://doi.org/10.1145/3154794 


\section{INTRODUCTION}

Private set intersection (PSI) allows two parties, $P_{1}$ and $P_{2}$, holding sets $X$ and $Y$, respectively, to identify the intersection $X \cap Y$ without revealing any information about elements that are not in the intersection. The basic PSI functionality can be used in applications where two parties want to perform JOIN operations over database tables that they must keep private, e.g., private lists of preferences, properties, or personal records of clients or patients. PSI was used in several research projects for privacy-preserving computation of functionalities such as relationship path discovery in social networks (Mezzour et al. 2009), botnet detection (Nagaraja et al. 2010), testing of fully sequenced human genomes (Baldi et al. 2011), proximity testing (Narayanan et al. 2011), or cheater detection in online games (Bursztein et al. 2011).

PSI has been a very active research field, and there have been many suggestions for PSI protocols. The large number of proposed protocols makes it non-trivial to perform comprehensive cross-evaluations. This is further complicated by the fact that many protocol designs have not been implemented and evaluated, were analyzed under different assumptions and observations, and were often optimized w.r.t. overall run-time while neglecting other relevant factors such as communication. Furthermore, even though several PSI protocols have been introduced, practical applications that need to compute the intersection of privacy-sensitive lists often use insecure solutions. The reason for the poor acceptance of secure solutions is, among others, the poor efficiency of existing schemes, which have more than two orders of magnitude more overhead than insecure solutions.

In this article, we give an overview on existing efficient PSI protocols, optimize exiting PSI protocols, and describe a new PSI protocol based on efficient oblivious transfer (OT) extensions. We compare both the theoretical and empirical performance of all protocols on the same platform and evaluate their cost compared to the insecure hash-based solution used in practice. We show that our new PSI protocol achieves a reasonable overhead compared to solutions used in practice.

\subsection{Motivating Applications}

The motivation for our work comes from several practical applications that require the PSI functionality. In the following, we list three of these applications:

Measuring Ad Conversion Rates. Measuring ad conversion rates is done by comparing the list of people who have seen an ad with those who have completed a transaction. These lists are held by the advertiser (say, Google or Facebook), and by merchants, respectively. It is often possible to identify users on both ends, using identifiers such as credit card numbers, email addresses, and the like. A simple solution, which ignores privacy, is for one side to disclose its list of customers to the other side, which then computes the necessary statistics. Another option is to run a PSI protocol between the two parties. (The protocol should probably be a variant of PSI, e.g., compute total revenues from customers who have seen an ad. Such protocols can be derived from basic PSI protocols.) In fact, Facebook is running a service of this type with Datalogix, Epsilon, and Acxiom, companies which have transaction records for a large part of loyalty card holders in the U.S. According to reports, ${ }^{1}$ the computation is done using a variant of the insecure naïve hashing PSI protocol that we describe in Section 1.2. Our results show that it can be computed using secure protocols even for large data sets.

Security Incident Information Sharing. Security incident handlers can benefit from information sharing since it provides them with a global view during incidents. However, incident data is often

\footnotetext{
${ }_{1}^{1}$ See, e.g., https://www.eff.org/deeplinks/2012/09/deep-dive-facebook-and-datalogix-whats-actually-getting-shared-andhow-you-can-opt.
} 
sensitive and potentially embarrassing. The shared information might reveal information about the business of the company that provided it, or of its customers. Therefore, information is typically shared rather sparsely and protected using legal agreements. Automated large-scale sharing will improve security, and there is, in fact, work to that end, such as the IETF Managed Incident Lightweight Exchange (MILE) effort. Many computations that are applied to the shared data compute the intersection and its variants. Applying PSI to perform these computations can simplify the legal issues of information sharing. Efficient PSI protocols will enable it to be run often and in large scale.

Private Contact Discovery. When a new user registers to a service it is often essential to identify current registered users who are also contacts of the new user. This operation can be done by simply revealing the user's contact list to the service, but can also be done in a privacy preserving manner by running a PSI protocol between the user's contact list and the registered users of the service. This latter approach is used by the Signal and Secret applications, but for performance reasons they use the insecure naïve hashing PSI protocol. ${ }^{2}$ Signal is planning to use a solution based on Intel SGX for scalability reasons as proposed in Tamrakar et al. (2017). ${ }^{3}$

In these cases, each user has a small number of records $n_{2}$, e.g., $n_{2}=256$, whereas the service has millions of registered users (in our experiments we use $n_{1}=16,777,216$ ). It therefore holds that $n_{1} \gg n_{2}$. In our best PSI protocol, the client needs only $\mathrm{O}\left(n_{2} \log n_{1}\right)$ memory, $\mathrm{O}\left(n_{2}\right)$ symmetric cryptographic operations, and $\mathrm{O}\left(n_{1}\right)$ cheap hash table lookups, and the communication is $\mathrm{O}\left(n_{1} \log n_{1}\right)$. (The communication overhead is indeed high as it depends on $n_{1}$, but this seems inevitable if brute force searches are to be prevented.)

\subsection{Classification of PSI Protocols}

Securely intersecting two sets without leaking any information except the result of the intersection is one of the most prominent problems in secure computation. Several techniques have been proposed that realize the PSI functionality, such as the efficient but insecure naïve hashing solution, protocols that require a semi-trusted third party, or two-party PSI protocols. The earliest proposed two-party PSI protocols were special-purpose solutions based on public-key cryptography. Later, solutions were proposed using circuit-based generic techniques for secure computation that are mostly based on symmetric cryptography. The most recent developments are PSI protocols that are based on OT alone, and combine the efficiency of symmetric cryptographic primitives with special purpose optimizations. PSI protocols have been implemented and evaluated on smartphones (Huang et al. 2011; Asokan et al. 2013; Kiss et al. 2017).

A Naïve Solution. When confronted with the PSI problem, most novices come up with a solution where both parties apply a cryptographic hash function to their inputs and compare these hashes. Although this protocol is very efficient, it is insecure if the input domain is small or has low entropy, since one party could easily run a brute force attack that applies the hash function to all items that are likely to be in the input set and compare the results to the received hashes. (When inputs to PSI have high entropy, a protocol that compares hashes of the inputs can be used (Nagy et al. 2013).)

Third Party-Based PSI. Several PSI protocols have been proposed that utilize additional parties, e.g., Baldwin and Gramlich (1985). In Hazay and Lindell (2008), a trusted hardware token is used to evaluate an oblivious pseudo-random function (OPRF). This approach was extended to multiple untrusted hardware tokens in Fischlin et al. (2011). Several efficient server-aided protocols for PSI, which use a third party that holds no inputs, receives no outputs, and is assumed not to collaborate

\footnotetext{
${ }^{2}$ See https://whispersystems.org/blog/contact-discovery/ and https://medium.com/@davidbyttow/demystifying-secret12ab82fda29f, respectively.

${ }^{3}$ See https://signal.org/blog/private-contact-discovery/.
} 
with any of the parties were presented and benchmarked in Kamara et al. (2014). Outsourced PSI protocols, e.g., Abadi et al. $(2015,2017)$ and Oksuz et al. (2017), allow for reuse of encrypted datasets that are outsourced to the cloud, but are less efficient than Kamara et al. (2014) for a single run.

Public-Key-Based PSI. A PSI protocol based on Diffie-Hellmann (DH) key agreement was presented in Meadows (1986) (related ideas were presented in Shamir (1980) and Huberman et al. (1999)). Their protocol is based on the commutative properties of the $\mathrm{DH}$ function and was used for private preference matching, which allows two parties to verify if their preferences match to some degree.

Freedman et al. (2004) introduced PSI protocols secure against semi-honest and malicious adversaries in the standard model (rather than in the random oracle model (ROM) assumed in the $\mathrm{DH}$-based protocol). This protocol was based on polynomial interpolation, and was extended in Freedman et al. (2016), which presents protocols with simulation-based security against malicious adversaries, and evaluates the practical efficiency of the proposed hashing schemes. We discuss the proposed hashing schemes in Section 3. A similar approach that uses OPRFs to perform PSI was presented in Freedman et al. (2005). A protocol that uses polynomial interpolation and differentiation for finding intersections between multi-sets was presented in Kissner and Song (2005).

Another PSI protocol that uses public-key cryptography (more specifically, blind-RSA operations) and scales linearly in the number of elements was presented in Cristofaro and Tsudik (2010). In Debnath and Dutta (2015), a family of Bloom filter-based PSI protocols was introduced that realize PSI, PSI cardinality, and authenticated PSI functionalities. These protocols also use public-key operations, linear in the number of elements.

Circuit-Based PSI. Generic secure computation protocols have been subject to substantial efficiency improvements in the last decade. They allow the secure evaluation of arbitrary functions, expressed as arithmetic or Boolean circuits. Several Boolean circuits for PSI were proposed in Huang et al. (2012) and evaluated using the Yao's garbled circuits. The authors showed that their Java implementation scales very well with increasing security parameter and outperforms the blind-RSA protocol of Cristofaro and Tsudik (2010) for larger security parameter. We reflect on and present new optimizations for circuit-based PSI in Section 4.

OT-Based PSI. A Bloom-filter-based protocol PSI based on OT extension that achieves security against semi-honest and malicious adversaries has been given in Dong et al. (2013) and optimized for semi-honest adversaries in Pinkas et al. (2014). Recently in Lambæk (2016) and Rindal and Rosulek (2017a), it was shown that the Bloom filter-based protocol is insecure with respect to malicious adversaries. The authors of Rindal and Rosulek (2017a) showed how to fix the malicious secure Bloom filter-based protocol and gave the first implementation of a malicious secure PSI protocol, which computes the intersection of two sets with a million elements each in $\sim 200 \mathrm{~s}$.

In Rindal and Rosulek (2016), our OT-based PSI protocol of Pinkas et al. (2014) was extended to security against weakly malicious adversaries and used as a building block in a batch dualexecution Yao's garbled circuits protocol. In Lambæk (2016), our OT-based PSI protocol of Pinkas et al. (2015) was secured against a semi-honest $P_{1}$ and malicious $P_{2}$. An improved version of our OT-based PSI protocol in Pinkas et al. (2015) is given in Kolesnikov et al. (2016), which presents an efficient construction of an OPRF using the OT extension protocol of Kolesnikov and Kumaresan (2013) (cf. Section 2.2.3). The main observation of the authors is that the Kolesnikov and Kumaresan (2013) OT extension does not require an error correcting code but can instead use a pseudo-random code, which can be generated from a pseudo-random generator. The authors then apply their efficient OPRF construction to our Pinkas et al. (2015) protocol to greatly reduce the communication in the OTs, which is equal to the code length. In particular, the authors show that their construction achieves performance independent of the bit-length of elements $\sigma$. The OPRF construction 
Table 1. Bit-Length of the Underlying Codes (Equal to the Communication per OT in Bit) for Different Values of $\sigma-\log _{2}(n)$

\begin{tabular}{|l||c|c|c|c|c|c||c|}
\hline $\begin{array}{l}\text { Bit-Length } \\
\sigma-\log _{2}(\boldsymbol{n})\end{array}$ & $\mathbf{8}$ & $\mathbf{9}$ & $\mathbf{1 0}$ & $\mathbf{1 1}$ & $\mathbf{1 2}$ & $\mathbf{1 3}$ & Arbitrary \\
\hline \hline $\begin{array}{l}\text { Kolesnikov et al. } \\
\text { (2016) comm. per OT [bits] }\end{array}$ & \multicolumn{7}{|c|}{$424-448$ (depending on $n)$} \\
\hline Our comm. per OT [bits] & 255 & 256 & 264 & 268 & 270 & 271 & 512 \\
\hline
\end{tabular}

of Kolesnikov et al. (2016) is similar to our new OPRF construction described in Section 5. The idea of both works is to instantiate the OPRF that is implicitly used in the Pinkas et al. (2015) OTbased PSI protocol using larger codes. However, while Kolesnikov et al. (2016) replace the error correcting code with a pseudo-random code, we keep the error correcting code but use smaller, custom-tailored codes. We compare the code sizes for small values of $\sigma-\log _{2}(n)$ using the code table from Schürer and Schmid (2006) with the pseudo-random code size of Kolesnikov et al. (2016) in Table 1. While our instantiation requires less communication for small values of $\sigma-\log _{2}(n)$, it does not achieve performance independent of $\sigma$ and generating such a small custom-tailored code is a non-trivial problem. In the remainder of this article, we fix one error correcting code with bit-length 512 that supports all currently practical applications of PSI and which results in a communication overhead of factor $1.10 \times-1.15 \times$ for the full PSI protocol compared to Kolesnikov et al. (2016). Overall, we view our work as complementary to the work of Kolesnikov et al. (2016), where one can instantiate the underlying code based on the parameters to achieve the best overall performance. In fact, it has been shown in another independent and parallel work (Orrù et al. 2017) that our method of instantiating the underlying code enables an extension of the PSI protocol to malicious receivers, which does not work for the instantiation method of Kolesnikov et al. (2016). Finally, note that the work of Kolesnikov et al. (2016) and Orrù et al. (2017) also benefit from our improved hashing analysis of Section 3.

\subsection{Our Contributions}

We survey existing PSI protocols with security against semi-honest adversaries and solutions with a trusted third party. We then describe in detail the semi-honest secure PSI protocols and improve the performance of some protocols using carefully analyzed features of OT extension. We introduce a new OT-based PSI protocol, perform a detailed experimental comparison of the most promising semi-honest secure PSI protocols, and evaluate their overhead compared to the insecure naïve hashing protocol that is currently used in real-world applications. Our contributions are as follows.

Concrete Parameter Estimation for Hashing. In Freedman et al. (2004) the use of hashing-to-bins was suggested in the context of PSI to reduce the overhead for pairwise-comparisons. However, their analysis of the involved parameters was only asymptotic. In Section 3, we empirically analyze the hashing-to-bins techniques that were suggested in Freedman et al. (2004) and identify concrete parameters for the schemes. In addition, in Section 3.3 we utilize the permutation-based hashing techniques of Arbitman et al. (2010) to reduce the bit-length of the representations that are stored in the bins. This improves the performance of PSI protocols that require an overhead linear in the bit-length of elements, e.g., the protocols in Section 4.2 and Section 5.

Optimizations of Existing Protocols. We improve the circuit protocols using recent optimizations for OT extension (Asharov et al. 2013). In particular, in Section 4 we evaluate the circuit-based solution of Huang et al. (2012) on a secure evaluation of the GMW protocol, and utilize features of random OT (cf. Section 2.2) to optimize the performance of multiplexer gates (which form about 
Table 2. Runtime and Transferred Data for Private Set Intersection Protocols on Sets with $2^{20} \sigma=32$-Bit Elements and 128-Bit Security with a Single Thread over Gigabit LAN

\begin{tabular}{|c|c|c|c|c|c|}
\hline PSI Protocol & Hashing & $\begin{array}{c}\text { Server-Aided } \\
\text { (Kamara et al. } \\
2014) \\
\end{array}$ & $\begin{array}{c}\text { Public Key } \\
\text { (Meadows } \\
\text { 1986) } \\
\end{array}$ & $\begin{array}{c}\text { Circuit } \\
\text { PWC Section 4.2/ } \\
\text { OPRF Section 4.3 } \\
\end{array}$ & $\begin{array}{c}\text { OT+Hashing } \\
\text { Section } 3+ \\
\text { Section } 5 \\
\end{array}$ \\
\hline \multicolumn{6}{|c|}{ Equal set sizes $n_{1}=n_{2}=2^{20}$} \\
\hline Runtime(s) & 0.7 & 1.3 & 818.3 & 124.7 & 5.8 \\
\hline Comm. (MB) & 10 & 20 & 74 & 14,014 & 111 \\
\hline \multicolumn{6}{|c|}{ Unequal set sizes $2^{24}=n_{1} \gg n_{2}=2^{12}$} \\
\hline Runtime(s) & 6.1 & 7.6 & $12,712.3$ & 7.3 & 42.6 \\
\hline Comm. (MB) & 160 & 160 & 593 & 947 & 480 \\
\hline
\end{tabular}

two thirds of the circuit). Furthermore, in Section 4.2 we outline how to use the permutation-based hashing techniques to improve the performance of circuit-based PSI even further.

A Novel OT-Based PSI Protocol. We present a new PSI protocol that is based on OT (Section 5) and directly benefits from improvements in efficient OT extensions (Kolesnikov and Kumaresan 2013; Asharov et al. 2013). Our PSI protocol uses an efficient OPRF that is instantiated based on the $\left(\begin{array}{c}N \\ 1\end{array}\right)$-OT extension protocol of Kolesnikov and Kumaresan (2013) and uses the hashing techniques from Section 3 to reduce the communication overhead from $O\left(n^{2}\right)$ to $O(n)$. The resulting protocol has very low computation complexity since it mostly requires symmetric key operations and has even less communication than some public-key-based PSI protocols, which had the lowest communication before.

A Detailed Comparison of PSI Protocols. We implement the most promising SI protocols using state-of-the-art cryptographic techniques and compare their performance on one platform. Our implementations are available as open source at http://encrypto.de/code/JournalPSI. As far as we know, this is the first time that such a wide comparison has been made, since previous comparisons were either theoretical, compared implementations on different platforms or programming languages, or used implementations without state-of-the-art optimizations. Our implementations and experiments are described in detail in Section 6. Certain experimental results were unexpected. We give a partial summary of our results in Table 2 . We briefly describe our most prominent findings next.

- The Diffie-Hellman-based protocol (Meadows 1986), which was the first PSI protocol, is actually the most efficient w.r.t. communication (when implemented using elliptic-curve crypto). Therefore, it is suitable for settings with distant parties which have strong computation capabilities but limited connectivity.

- Generic circuit-based protocols (Huang et al. 2012) are less efficient than the newer, OTbased constructions, but they are more flexible and can easily be adapted for computing variants of the set intersection functionality (e.g., computing whether the size of the intersection exceeds some threshold). Our experiments also support the claim of Huang et al. (2012) that circuit-based PSI protocols are faster than the blind-RSA-based PSI protocol of Cristofaro and Tsudik (2010) for larger security parameters and given sufficient bandwidth.

- Compared to the insecure naïve hashing solution, previous PSI protocols are at least two orders of magnitude less efficient in runtime or communication. Our OT-based PSI protocol reduces this overhead to only one order of magnitude in both runtime and communication. 
-When intersecting sets with unequal sizes $\left(n_{1} \gg n_{2}\right)$, the runtime difference between most protocols remains similar to the runtime difference for equal set sizes $\left(n_{1}=n_{2}\right)$. The only exception is the newly added circuit-based OPRF protocol (Section 4.3), which achieves similar performance as the naïve hashing and server-aided solutions for unequal set sizes.

\subsection{Additions to Conference Versions}

This article is a significantly extended and improved version of the conference papers (Pinkas et al. 2014) and Pinkas et al. (2015). Compared to these papers, we add the following contributions:

Broader Scope. We broadened the scope of the work by describing and benchmarking the circuitbased OPRF protocol of Pinkas et al. (2009) in Section 4.3.

Extended Hashing Parameter Analysis. We extend the hashing parameter analysis for schemes that are using pairwise comparison. In our previous work, we only bounded the hashing failure for one particular set of parameters that is tailored to one use-case. However, the hashing parameters for which PSI protocols perform well change depending on the settings (unequal set sizes, different networks, and so on). We show a trade-off between different parameters, resulting in a large variety of parameters that perform well for different settings.

Optimizations. In previous work, our OT-based PSI protocol scaled linear in the bit-length of the inputs, which decreased its performance on arbitrary input data. We now outline how to achieve performance independent of the bit-length in Section 5 by using more efficient instantiations of underlying primitives (cf. Section 2.2.3).

Unequal Set Sizes. We extend the focus of the work to unequal set sizes where $n_{1} \gg n_{2}$. This setting is relevant for use-cases where, e.g., an end user wants to compare its data (few hundred elements) to a company's database (several million elements). We show how to modify the circuitbased protocols (Section 4.3) as well as our OT-based protocol (Section 3.2.2) to efficiently extend to this setting, and perform experiments for the protocols (Section 6.2.3).

Scalability. The largest sets on which secure two-party PSI protocols were evaluated until now were of size $2^{24}$ (Pinkas et al. 2015). We demonstrate the scalability of our novel OT-based PSI protocol by processing two sets of a billion $\sigma=128$-bit elements each (Section 6.2.4).

\section{PRELIMINARIES}

We give our notation and security definitions in Section 2.1, review recent relevant work on OT in Section 2.2, and outline how to hash large inputs into smaller domains in Section 2.3.

\subsection{Notation and Security Definitions}

We denote the parties as $P_{1}$ and $P_{2}$, and their respective input sets as $X$ and $Y$ with $|X|=n_{1}$ and $|Y|=n_{2}$. We refer to elements from $X$ as $X$ and elements from $Y$ as $y$ and each element has bitlength $\sigma$ (cf. Section 2.3 for the relation between $n$ and $\sigma$ ). We write $b[i]$ for the $i$-th element of a list $b$, denote the bitwise-AND between two bit strings $a$ and $b$ of equal length as $a \wedge b$ and the bitwise-XOR as $a \oplus b$. We denote a constant string of $m$ zeros (or ones) as $0^{m}$ (or $1^{m}$ ). We use a correlation robust function (CRF) (cf. Section 2.1), a pseudo-random generator (PRG), a pseudorandom permutation (PRP), and an OPRF (see definitions below). We write $\left(\begin{array}{c}N \\ 1\end{array}\right)$-OT $\mathrm{OT}_{\ell}^{m}$ for $m$ parallel 1-out-of- $N$ OTs on $\ell$-bit strings, and write $\mathrm{OT}_{\ell}^{m}$ for $\left(\begin{array}{l}2 \\ 1\end{array}\right)-\mathrm{OT}_{\ell}^{m}$. In a similar fashion, we denote the random OT functionality (cf. Section 2.2.2), where the functionality chooses $m N$-tuples of random $\ell$-bit strings as $\left(\begin{array}{c}N \\ 1\end{array}\right)-\mathrm{ROT}_{\ell}^{m}$. We fix the key sizes to a 128-bit security level according to the NIST guidelines (NIST 2012): symmetric security parameter $\kappa=128$, asymmetric security parameter $\rho=3,072$, statistical security parameter $\lambda=40$, and elliptic curve size $\varphi=284$ for Koblitz curve 
K-283 when using point compression (this is the number of bits for one coordinate and a sign-bit). We denote and fix the hashing failure parameter, which affects the correctness of some protocols as $\eta=40$, meaning that hashing failures occur with probability $<2^{-40}$.

Adversary Definition. The secure computation literature considers two types of adversaries with different strengths: A semi-honest adversary tries to learn as much information as possible from a given protocol execution but is not able to deviate from the protocol steps. The semi-honest adversary model is appropriate for scenarios where execution of the intended software is guaranteed via software attestation or where an untrusted third party is able to obtain the transcript of the protocol after its execution, either by stealing it or by legally enforcing its disclosure. The stronger, malicious adversary extends the semi-honest adversary by being able to deviate arbitrarily from the protocol.

Most protocols for PSI, as well as this article, focus on solutions that are secure against semihonest adversaries. PSI protocols for the malicious setting exist, but they are less efficient than protocols for the semi-honest setting (see, e.g., Freedman et al. (2004), Cristofaro et al. (2010), and Rindal and Rosulek (2016, 2017a, 2017b)). Currently, the fastest protocol with malicious security (Rindal and Rosulek 2017b) is only 3x slower than the semi-honest protocol of Kolesnikov et al. (2016). We also note that circuit-based PSI protocols that are evaluated with Yao's protocol can efficiently be secured against a malicious client by using OT extension with malicious security (e.g., Asharov et al. (2015)), which adds very little overhead: the OPRF-based protocol directly and the SCS-based protocol requires us to add a small circuit that makes sure that the inputs are sorted.

Security Definition. Following the definitions of Goldreich (2004), a protocol is secure if whatever can be computed by a party participating in the protocol can be computed based only on the input and output of this party. Namely, the actual messages delivered during the protocol provide no additional information beyond the input and output. This definition is formalized based on the simulation paradigm: it is required that a party's view in a protocol execution (namely, all messages sent and received by the party in the protocol) can be simulated given only its input and output. We note that in the case of semi-honest adversaries, and a protocol computing deterministic functionalities (as is the intersection functionality), it suffices that the simulator outputs the view of the party that is controlled by the adversary. See Goldreich (2004) for the detailed definition of security.

The Random Oracle Model (ROM). As most previous works on efficient PSI, we use the ROM to achieve more efficient implementations (Bellare and Rogaway 1993). The security of cryptographic constructions can be proven in the "standard model," where security is based on well-researched complexity assumptions, or in the ROM, which is based on modeling a hash function as a random function (Bellare and Rogaway 1993). There are many criticisms about the ROM and in the theory of cryptography proofs this model is considered heuristic. Yet, protocols in the ROM are often more efficient than protocols that are proven in the standard model.

The efficiency gain in using the ROM is particularly true with regards to protocols for PSI. The only semi-honest protocol that we describe that is in the standard model is the protocol based on oblivious polynomial evaluation by Freedman et al. (2004, 2016), but that protocol is less efficient than the other protocols that we present. The public-key-based protocols (based on Diffie-Hellman and blind RSA) use a hash function $H()$ that must be modeled as a random oracle, or modeled using another non-standard assumption. The other protocols (the generic protocol, as well as the protocol based on Bloom filters and the new OT-based protocol) can be implemented without a random oracle assumption, but to speedup the computation of OT in these protocols we must use random OT extension, whose efficient implementation relies on a function that must be modeled as a random oracle. 
Correlation Robustness. A CRF $H:\{0,1\}^{\kappa} \mapsto\{0,1\}^{\ell}$ is a function for which, given uniformly and randomly chosen $t_{1}, \ldots, t_{m}, s \in\{0,1\}^{\kappa}$, an adversary is unable to computationally distinguish $t_{1}, \ldots, t_{m}, H\left(t_{1} \oplus s\right), \ldots, H\left(t_{m} \oplus s\right)$ from a uniform distribution. It is a weaker assumption than the ROM and is used in OT extension as well as Yao's garbled circuit protocol. Traditionally, many implementations use a hash function (e.g., SHA) to increase the performance. An instantiation of the CRF in Yao's garbled circuit protocol that uses fixed-key AES and greatly improves performance was proposed in Bellare et al. (2013) and refined in Zahur et al. (2015) for use in the half-gates scheme. In this article, we use both optimizations.

Oblivious Pseudo-Random Functions. An OPRF (Freedman et al. 2005) is a function $F$ : $\left(\{0,1\}^{\kappa},\{0,1\}^{\sigma}\right) \mapsto\left(\perp,\{0,1\}^{\ell}\right)$ that, given a key $k$ from $P_{1}$ and an input element $e$ from $P_{2}$, computes and outputs $F_{k}(e)$ to $P_{2} . P_{1}$ obtains no output and learns no information about $e$ while $P_{2}$ learns no information about $k$. OPRFs can be used for PSI by first evaluating the OPRF protocol on the set of $P_{2}$ and then having $P_{1}$, who knows the secret key $k$, evaluate the OPRF locally on its own set, and send the OPRF output to $P_{2}$, who computes a plaintext intersection. There exist several instantiations for OPRFs, described in Freedman et al. (2005): based on generic secure computation techniques (using an AES circuit (Pinkas et al. 2009)), based on the Diffie-Hellman assumption, or based on OT. In Section 4.3 we analyze the efficiency of generic secure computation-based OPRF instantiations. In Section 5 we give a more efficient OT-based instantiation based on the $\left(\begin{array}{c}N \\ 1\end{array}\right)$-ROT protocol of Kolesnikov and Kumaresan (2013).

\subsection{Oblivious Transfer}

OT is a major building block for secure computation. When executing $m$ invocations of 1-outof-2 OT on $\ell$-bit strings (denoted $\left(\begin{array}{c}2 \\ 1\end{array}\right)$-OT $\left.{ }_{\ell}^{m}\right)$, the sender $S$ holds $m$ message pairs $\left(x_{0}^{i}, x_{1}^{i}\right)$ with $x_{0}^{i}, x_{1}^{i} \in\{0,1\}^{\ell}$, while the receiver $R$ holds an $m$-bit choice vector $b$. At the end of the protocol, $R$ receives $x_{b[i]}^{i}$ but learns nothing about $x_{1-b[i]}^{i}$, and $S$ learns nothing about $b$. Many OT protocols have been proposed, most notably (for the semi-honest model) the Naor-Pinkas OT (Naor and Pinkas 2001), which uses public-key operations and has amortized complexity of $3 m$ public-key operations when performing $m$ OTs.

OT extension (Beaver 1996; Ishai et al. 2003) reduces the number of expensive public-key operations for $\mathrm{OT}_{\ell}^{m}$ to that of only $\mathrm{OT}_{\kappa}^{\kappa}$, and computes the rest of the protocol using more efficient symmetric cryptographic operations that are faster by orders of magnitude. The security parameter $\kappa$ is essentially independent of the number of OTs $m$, and can be as small as 128 . Thereby, the computational complexity for performing OT is reduced to such an extent that the network bandwidth becomes the main bottleneck (Asharov et al. 2013).

Recently, the efficiency of OT extension protocols has gained a lot of attention. In Kolesnikov and Kumaresan (2013), an efficient $\left(\begin{array}{c}N \\ 1\end{array}\right)$-OT extension protocol was shown, that has sub-linear communication in $\kappa$ for short messages. Another protocol improvement is outlined in Asharov et al. (2013) and Kolesnikov and Kumaresan (2013), which decreases the communication from $R$ to $S$ by half. Additionally, several works (Asharov et al. 2013; Nielsen et al. 2012) improve the efficiency of OT by using an OT variant, called random OT. In random OT, $\left(x_{0}^{i}, x_{1}^{i}\right)$ are chosen uniformly and randomly within the OT and are output to $S$, thereby removing the final message from $S$ to $R$. Random OT is useful for many applications, and we show how it can reduce the overhead of PSI.

We describe the OT extension protocol of Asharov et al. (2013) and Ishai et al. (2003), the random OT functionality, and the $\left(\begin{array}{c}N \\ 1\end{array}\right)$-OT extension protocol of Kolesnikov and Kumaresan (2013) in more detail next. 
2.2.1 1-out-of-2 OT Extension. (Ishai et al. 2003) describe a $\left(\begin{array}{l}2 \\ 1\end{array}\right)$-OT extension protocol that extends $\mathrm{OT}_{\kappa}^{\kappa}$ ( $\kappa$ OTs of $\kappa$ bits) to OT $\mathrm{OT}_{\ell}^{m}(m$ OTs of $\ell$ bits). We describe the OT extension protocol of Ishai et al. (2003) with optimizations of Asharov et al. (2013) in Prot. 1.

PROTOCOL 1 (OT Extension Protocol of Ishai et AL. (2003)).

- Input of $P_{1}: m$ pairs of $\ell$-bit strings $\left(x_{0}^{j}, x_{1}^{j}\right), 1 \leq j \leq m$.

- Input of $P_{2}$ : Choice vector $b \in\{0,1\}^{m}$.

- Common Input: Symmetric security parameter $\kappa$ and number of base-OTs $\beta=\kappa$.

- Oracles and cryptographic primitives:Both parties have access to an $\mathrm{OT}_{\kappa}^{\beta}$ oracle, a PRG $G:\{0,1\}^{\kappa} \mapsto\{0,1\}^{m}$, and a CRF $H:\{0,1\}^{\beta} \mapsto\{0,1\}^{\ell}$.

(1) $P_{1}$ initializes a random vector $s \in\{0,1\}^{\beta}$ and $P_{2}$ chooses $\beta$ random key pairs $\left(k_{0}^{i}, k_{1}^{i}\right) \in\{0,1\}^{2 \kappa}$, for $1 \leq i \leq \beta$.

(2) The parties invoke the $\mathrm{OT}_{\kappa}^{\beta}$ oracle where, in the $i$-th OT, $P_{1}$ plays the receiver with input $s[i]$ and $P_{2}$ plays the sender with inputs $\left(k_{0}^{i}, k_{1}^{i}\right)$.

(3) $P_{2}$ computes $t^{i}=G\left(k_{i}^{0}\right)$ and $u^{i}=t^{i} \oplus G\left(k_{i}^{1}\right) \oplus b$, and sends $u^{i}$ to $P_{1}$, for $1 \leq i \leq \beta$. Let $T=\left[t^{1}|\ldots| t^{\beta}\right]$ denote a random $m \times \beta$ bit matrix that is generated by $P_{2}$ where the $i$-th column is $t^{i}$ and the $j$-th row is $t_{j}$, for $1 \leq i \leq \beta$ and $1 \leq j \leq m$.

(4) $P_{1}$ defines $q^{i}=\left(s[i] \cdot u^{i}\right) \oplus G\left(k_{i}^{s[i]}\right)$ (note that $q^{i}=\left((s[i] \cdot b) \oplus t^{i}\right)$.

(5) Let $Q=\left[q^{1}|\ldots| q^{\beta}\right]$ denote the $m \times \beta$ bit matrix where the $i$-th column is $q^{i}$. Let $q_{j}$ denote the $j$-th row of the matrix $Q$ (note that $q_{j}=(b[j] \cdot s) \oplus t_{j}$.

(6) $P_{1}$ sends $\left(y_{j}^{0}, y_{j}^{1}\right)$ for every $1 \leq j \leq m$, where

$$
y_{j}^{0}=x_{j}^{0} \oplus H\left(q_{j}\right) \text { and } y_{j}^{1}=x_{j}^{1} \oplus H\left(q_{j} \oplus s\right)
$$

(7) $P_{2}$ computes $x_{j}^{b[j]}=y_{j}^{b[j]} \oplus H\left(t_{j}\right)$, for $1 \leq j \leq m$.

(8) Output: $P_{1}$ has no output; $P_{2}$ outputs $\left(x_{b[1]}^{1}, \ldots, x_{b[m]}^{m}\right)$.

Efficiency. Overall, when using OT extension, the sender in $\mathrm{OT}_{\ell}^{m}$ has to evaluate $2 m$ CRFs and $m$ PRGs, and send $2 m \ell$ bits, while the receiver has to evaluate $m$ CRFs and $2 m$ PRGs, and send $m \kappa$ bits. (In addition, there is a preprocessing cost of OT ${ }_{\kappa}^{\kappa}$ public-key-based OTs, which is negligible compared to the main protocol if $\kappa \ll m$.)

2.2.2 Random OT Extension. To improve efficiency of OT extension protocols in specific settings, several works (Nielsen et al. 2012; Asharov et al. 2013) use a special purpose OT functionality, called random OT. In a random OT, $\left(x_{0}^{i}, x_{1}^{i}\right)$ are chosen uniformly and randomly during the OT and are output to $P_{1}$. A random OT extension protocol can be obtained by leaving out the last message from $P_{1}$ to $P_{2}$, containing $\left(y_{0}^{i}, y_{1}^{i}\right)$. In more detail, $P_{1}$ has no input to the protocol and sets $\left(x_{0}^{j}=H\left(q_{j}\right), x_{1}^{j}=H\left(q_{j} \oplus s\right)\right)$ in Step 6 in Prot. 1 while $P_{2}$ sets $x_{b[j]}^{i}=H\left(t_{j}\right)$ in Step 7. $P_{1}$ then outputs $m$ pairs of $\ell$-bit strings $\left(x_{0}^{j}, x_{1}^{j}\right)$ and $P_{2}$ outputs $x_{b[j]}^{j}$. This random OT extension protocol reduces the bits that $P_{1}$ has to send from $2 m \ell$ to 0 at the expense of the stronger assumption that $H$ is modeled as a Random Oracle Model (ROM) instead of a CRF.

2.2.3 1-out-of-N OT Extension. In Kolesnikov and Kumaresan (2013), an efficient $\left(\begin{array}{c}N \\ 1\end{array}\right)$-OT extension protocol was introduced that allows for transfer of short messages with sublinear communication in $\kappa$. The protocol builds on the original OT extension protocol of Ishai et al. (2003) 
and encodes the choices of $R$ using an error correcting code $C^{N}=c_{0}, \ldots, c_{N-1}$, which encodes $\left\lceil\log _{2} N\right\rceil$-bit words with $p$-bit codewords that have at least $\kappa$ Hamming distance from each other. We highlight the differences of the Kolesnikov and Kumaresan (2013) $\left(\begin{array}{c}N \\ 1\end{array}\right)$-OT compared to the Ishai et al. (2003) $\left(\begin{array}{l}2 \\ 1\end{array}\right)$-OT in Prot. 2.

PROTOCOL 2 (Differences of 1-out-of-N over 1-out-of-2 OT Extension in Prot. 1).

- Input of $P_{1}: m N$-tuples of $\ell$-bit strings $\left(x_{0}^{j}, \ldots, x_{N-1}^{j}\right), 1 \leq j \leq m$.

- Input of $\boldsymbol{P}_{2}$ : Choice integers $b_{j} \in\{0, N-1\}, 1 \leq j \leq m$.

- Common Input: Symmetric security parameter $\kappa, \beta=2 \kappa$, and error correcting code $C^{N}=c_{0}, \ldots, c_{N-1}$.

(3) $P_{2}$ computes $t^{i}=G\left(k_{i}^{0}\right), u^{i}=G\left(k_{i}^{1}\right), v_{j}=t_{j} \oplus u_{j} \oplus c_{b_{j}}$, and sends $v^{i}$ to $P_{1}$, for $1 \leq i \leq$ $\beta$ and $1 \leq j \leq m$. Note that $T=\left[t^{1}|\ldots| t^{\beta}\right]$ denotes a random $m \times \beta$ bit matrix where the $i$-th column is $t^{i}$ and the $j$-th row is $t_{j}$ (the same holds for $U=\left[u^{1}|\ldots| u^{\beta}\right]$ and $\left.V=\left[v^{1}|\ldots| v^{\beta}\right]\right)$.

(4) $P_{1}$ defines $q^{i}=\left(s[i] \cdot v^{i}\right) \oplus G\left(k_{i}^{s[i]}\right)$ (note that $\left.q_{j}=\left(s \wedge c_{b_{j}}\right) \oplus t_{j}\right)$.

(5) For each $0 \leq w<N-1$ and $1 \leq j \leq m, P_{1}$ computes and sends

$$
y_{j}^{w}=x_{j}^{w} \oplus H\left(q_{j} \oplus\left(s \wedge c_{w}\right)\right)
$$

(8) Output: $P_{1}$ has no output; $P_{2}$ outputs $\left(x_{b_{1}}^{1}, \ldots, x_{b_{m}}^{m}\right)$.

Two things are noteworthy in this $\left(\begin{array}{c}N \\ 1\end{array}\right)$-OT extension protocol. First, we can also use the random OT extension functionality by having $S$ set $x_{w}^{i}=H\left(q_{i} \oplus\left(s \wedge c_{w}\right)\right)$ and $R$ set $x_{b[i]}^{i}=H\left(t_{i}\right)$. Second, to achieve the same computational security level $\kappa=128$ as in the original $\left(\begin{array}{l}2 \\ 1\end{array}\right)$-OT extension protocol of Ishai et al. (2003), the parties have to increase the number of base-OTs $\beta$ to the codeword length $p$ of the underlying code, which depends on $N$ (cf. Kolesnikov and Kumaresan (2013)). The reason for the increase in base OTs is that the Hamming distance between the codewords has to be at least $\kappa$. For $2<N \leq 2 \kappa$, Kolesnikov and Kumaresan (2013) proposes to use the Walsh-Hadamard code, which encodes up to $2 \kappa$ words to codewords of length $2 \kappa$ with relative Hamming distance $\kappa$. However, in our OT-based PSI protocol, we use $\sigma$-bit elements as input to the $\left(\begin{array}{c}N \\ 1\end{array}\right)$-OT protocol of Kolesnikov and Kumaresan (2013) and hence need to handle $2^{\sigma}=N \gg 2 \kappa$. To process such a $\sigma$-bit element, we need to find an error correcting code that processes $2^{\sigma}$ input elements with codewords of relative Hamming distance 128-bit and short codesize. As an example, when processing $\sigma=13$-bit elements, we could use a code of size 271-bit, as given in Schürer and Schmid (2006).

In the remainder of the article and for ease of presentation, we fix a linear $\mathrm{BCH}$ code, generated from Morelos-Zaragoza (2006), which encodes up to $2^{77}$ words to codewords of length 512 with relative Hamming distance $\kappa$, which is denoted as a $\left[2^{77}, 512,129\right]$ code. Using the permutationbased hashing techniques outlined in Section 3.3, and assuming a statistical security of $\lambda=40$ bit, this allows us to process sets with up to 100 billion $\left(2^{37}\right)$ elements independently of their bitlength $\sigma$, which suffices for most applications.

Efficiency. Evaluating one $\left(\begin{array}{c}N \\ 1\end{array}\right)$-ROT using the $\left(\begin{array}{c}N \\ 1\end{array}\right)$-OT extension protocol of Kolesnikov and Kumaresan (2013) and our linear $\mathrm{BCH}$ code requires 512 bits of communication and $N$ CRF evaluations. Note that, although the high number of CRF evaluations for the $\left(\begin{array}{c}N \\ 1\end{array}\right)$-OT seems prohibitive for large $N$, we only need to perform a constant number (say three) of CRF evaluations in our protocol. In comparison, naïvely building $\left(\begin{array}{c}N \\ 1\end{array}\right)$-ROT from $\left(\begin{array}{l}2 \\ 1\end{array}\right)$-OT extension would require 
$\log N\left(\begin{array}{l}2 \\ 1\end{array}\right)$-OT invocations and hence require $128 \log N$ bits of communication and $2 \log N$ CRF evaluations. More concretely, when computing the intersection between two million element sets using our OT-based PSI protocol in Section 5 , we would have $N \approx 2^{60}$ and hence would require 512-bit communication using the $\left(\begin{array}{c}N \\ 1\end{array}\right)$-OT extension protocol of Kolesnikov and Kumaresan (2013) and 7,680 bits communication using the regular $\left(\begin{array}{l}2 \\ 1\end{array}\right)$-OT extension protocol of Ishai et al. (2003) with most recent optimizations of Asharov et al. (2013) and Kolesnikov and Kumaresan (2013).

\subsection{Hashing Inputs to a Smaller Domain}

The performance of some PSI protocols depends on the length of the representation of their inputs. This is particularly true for protocols that run an OT for each bit of the input representation, e.g., the protocols described in Section 4.2 and Section 5.

When the original input representation is sparse, we can first use a hash function to map the identities of the input items to identities from a smaller domain with a shorter representation. We then run the original protocol on that representation, resulting in a more efficient execution. The size of the new domain should be large enough so that no two different input items are mapped to the same value. The theoretical analysis of this mapping, related to the birthday paradox, shows that when $n$ items are mapped to a domain of size $D$ using a random hash function, the probability of experiencing a collision is $p=1-e^{-n \cdot(n-1) /(2 D)}$, and can be approximated as $p \approx n^{2} /(2 D)$ (see Motwani and Raghavan $(1995,45)$. Let us denote the length of the representation of items in $D$ as $d=\log D$. Then $p \approx n^{2} /\left(2 \cdot 2^{d}\right)$, and therefore

$$
d=2 \log (n)-1-\log (p) .
$$

\section{HASHING SCHEMES AND PSI}

Computing the plaintext intersection between two sets is often done using hashing techniques. The parties agree on a publicly known random hash function to map elements to a hash table, which consists of multiple bins. If an input element is in the intersection, both parties map it to the same bin. Hence, the parties only need to compare the elements that are in the same bin to identify intersecting elements. Thereby, the average number of comparisons between elements can be reduced from $O\left(n^{2}\right)$ to $O(n)$ for pairwise comparisons.

In a similar fashion, PSI protocols that privately compute the equality between values can use hashing techniques to reduce the number of comparisons (Freedman et al. 2004, 2016). Examples for such private equality test protocols are Freedman et al. (2004), Huang et al. (2012), and Carter et al. (2013), the circuit-based protocol in Section 4.2 or our OT-based protocol in Section 5. When naïvely using hashing techniques, if $n$ items are mapped to $n$ bins, then the average number of items in a bin is $O(1)$, checking for an intersection in a bin takes $O(1)$ work, and hence the total number of comparisons is $O(n)$. However, privacy requires that the parties hide from each other how many of their inputs were mapped to each bin. ${ }^{4}$ As a result, we must calculate in advance the number of items that will be mapped to the most populated bin (with high probability), and then set all bins to be of that size. (This can be done by storing dummy items in bins which are not fully occupied.) This change hides the bin sizes but also increases the overhead of the protocol, since the number of comparisons per bin now depends on the size of the most populated bin (worst case) rather than on the actual number of items in the bin (average case).

\footnotetext{
${ }^{4}$ Otherwise, and since the hash function is public, some information is leaked about the input. For example, if no items of $P_{1}$ were mapped to the first bin by the hash function $h$, then $P_{2}$ learns that $P_{1}$ has no inputs in the set $h^{-1}(1)$, which covers about $1 / n$ of the input range.
} 
In fact, this worst case analysis is key to balancing security and efficiency. On one hand, if the estimation is too optimistic, the probability of a party failing to perform the mapping becomes intolerable. As a result, the output might be inaccurate (since not all items can be mapped to bins), or one party needs to request a new hash function (a request that leaks information about the input set of that party). On the other hand, the number of performed comparisons and hence the protocol overhead can become prohibitive if the analysis is too pessimistic. The work of Freedman et al. $(2004,2016)$ gave asymptotic values for this analysis and of the resulting overhead. They left the task of setting appropriate parameters for the hashing schemes to future work.

In this section, we revisit the simple hashing (Section 3.1) and cuckoo hashing (Section 3.2) schemes, used in Freedman et al. $(2004,2016)$. We describe how to use both hashing schemes in the context of PSI and give a concrete parameter analysis that balances security and efficiency. Finally, we show how the bit-length of the representations that are stored in the bins can be reduced using permutation-based mapping, which improves the performance of some PSI protocols (Section 3.3).

Note that, for our hashing failure analysis, we use a dedicated hashing failure parameter $\eta$, which is different from the statistical security parameter $\lambda$. We use a dedicated parameter since our analysis requires running empirical experiments for determining concrete numbers, which would have cost several hundred thousand USD for $2^{40}$ iterations in the Amazon EC2 cloud. Hence, we perform the experiments and give concrete numbers for $\eta=30$ and interpolate from these results to $\eta=40$.

\subsection{Simple Hashing}

In the simplest hashing scheme, the hash table consists of $b$ bins $B_{1} \ldots B_{b}$. Hashing is done by mapping each input element $e$ to a bin $B_{h(e)}$ using a hash function $h:\{0,1\}^{\sigma} \mapsto[1, b]$ that was chosen uniformly at random and independently of the input elements. An element is always added to the bin to which it is mapped, regardless of whether other elements are already stored in that bin.

3.1.1 Simple Hashing for PSI. To apply simple hashing in the context of PSI, both parties map their elements to $b$ bins. The intersection is then computed by having both parties separately compare the items mapped to bin $i \in[1, \ldots, b]$. To hide the number of elements that were mapped to a bin, the parties need to pad their bins using dummy elements to contain $\max _{b}$ elements. This $^{-}$ maximum bin size must ensure that, except with probability $<2^{-\eta}$, no bin will contain more than $\max _{b}$ real elements.

3.1.2 Simple Hashing Parameter Analysis. Estimating $\max _{b}$ has been subject to extensive research (Gonnet 1981; Raab and Steger 1998; Mitzenmacher 2001). When hashing $n$ elements to $b=n$ bins, Gonnet (1981) showed that $\max _{b}=\frac{\ln n}{\ln \ln n}(1+o(1))$ with high probability. In this case, there is a difference between the expected and the maximum number of elements mapped to a bin, which are 1 and $O\left(\frac{\ln n}{\ln \ln n}\right)$, respectively. Let us revisit the analysis of Motwani and Raghavan (1995) that analyzes the probability of the following event, " $n$ balls are mapped at random to $b$ bins, and the most occupied bin has at least $k$ balls":

$$
\begin{aligned}
P\left(\text { “ } \exists \text { bin with } \geq \max _{b}\right. \text { elements") } & \leq \sum_{i=1}^{b} P \text { ("bin } i \text { has } \geq \max _{b} \text { elements") } \\
& =b \cdot\left[P \text { ("bin } i \text { has } \geq \max _{b} \text { elements") }\right] \\
& =b \cdot\left[\left(\sum_{i=\max _{b}}^{n}\left(\begin{array}{l}
n \\
i
\end{array}\right) \cdot\left(\frac{1}{b}\right)^{i} \cdot\left(1-\frac{1}{b}\right)^{n-i}\right] .\right.
\end{aligned}
$$


Table 3. The Maximum Bin Sizes $\max _{b}$ Required to Ensure No Overflow Occurs When Mapping $n$ Items to $b=n$ Bins, According to Equation (3)

\begin{tabular}{|l||r|r|r|r|r||r|r|r|r|r|}
\hline Hash Failure Parameter $\boldsymbol{\eta}$ & \multicolumn{3}{|c||}{30} & \multicolumn{5}{|c|}{40} \\
\hline Set Size $\boldsymbol{n}$ & $2^{8}$ & $2^{12}$ & $2^{16}$ & $2^{20}$ & $2^{24}$ & $2^{8}$ & $2^{12}$ & $2^{16}$ & $2^{20}$ & $2^{24}$ \\
\hline Maximum Bin Size $\max _{\boldsymbol{b}}$ (Equation 3) & 13 & 15 & 16 & 17 & 18 & 16 & 17 & 18 & 19 & 20 \\
\hline
\end{tabular}

Table 4. The Maximum Bin Sizes $\max _{b}$ Required to Ensure No Overflow Occurs When Mapping $n=2 n_{1}$ Items to $b=2.4 n_{2}$ Bins for $n_{1} \gg n_{2}$, According to Equation (3)

\begin{tabular}{|c|c|c|c|c|c|c|}
\hline \multirow{2}{*}{$\begin{array}{l}\text { Set Size } n_{2} \\
\text { Set Size } n_{1} \\
\end{array}$} & \multicolumn{3}{|c|}{$2^{8}$} & \multicolumn{3}{|c|}{$2^{12}$} \\
\hline & $2^{16}$ & $2^{20}$ & $2^{24}$ & $2^{16}$ & $2^{20}$ & $2^{24}$ \\
\hline \multicolumn{7}{|l|}{ Hash Failure Parameter $\eta=30$} \\
\hline Maximum Bin Size $\max _{b}$ (Equation 3) & 323 & 3,825 & 56,196 & 48 & 329 & 3,852 \\
\hline \multicolumn{7}{|l|}{ Hash Failure Parameter $\eta=40$} \\
\hline Maximum Bin Size $\max _{b}$ (Equation 3) & 338 & 3,881 & 56,412 & 53 & 344 & 3,905 \\
\hline
\end{tabular}

Case $n=b$. Using GMP with 1,024-bit floating point precision, we calculate $\max _{b}$ when mapping $n \in\left\{2^{8}, 2^{12}, 2^{16}, 2^{20}, 2^{24}\right\}$ elements to $b=n$ bins using Equation (3), choose the minimal value of $\max _{b}$ that reduces the failure probability to below $2^{-30}$, and $2^{-40}$ and depict the results in Table 3.

Case $n \gg b$. In certain settings, the server $P_{1}$ has a much larger set than the client $P_{2}$. For simple hashing, this translates to the number of elements $n$ being much larger than the number of bins $b$. Later in the article, we perform experiments for this setting (cf. Section 6.2.3), where $P_{2}$ has a set of size $n_{2} \in\left\{2^{8}, 2^{12}\right\}$, while $P_{1}$ has a set of size $n_{1} \in\left\{2^{16}, 2^{20}, 2^{24}\right\}$ and both map $n=2 n_{1}$ elements into $b=2.4 n_{2}$ bins. To determine $\max _{b}$ in this setting, we evaluate Equation (3) with these set sizes and depict $\max _{b}$ for hashing failure probabilities $2^{-30}$ and $2^{-40}$ in Table 4. From the results, we can observe that as the fraction $n_{1} / n_{2}$ grows, the maximum number of bin grows closer to the expected number of bins.

\subsection{Cuckoo Hashing}

Cuckoo hashing (Pagh and Rodler 2001) uses $k$ hash functions $h_{1}, \ldots, h_{k}:\{0,1\}^{\sigma} \mapsto[1, b]$ to map $m$ elements to $b=\epsilon n$ bins. The scheme avoids collisions by relocating elements when a collision is found using the following procedure: An element $e$ is inserted into a bin $B_{h_{1}(e)}$. Any prior contents $o$ of $B_{h_{1}(e)}$ are evicted to a new bin $B_{h_{i}(o)}$, using $h_{i}$ to determine the new bin location, where $h_{i}(o) \neq$ $h_{1}(e)$ for $i \in[1 \ldots k]$. The procedure is repeated until no more evictions are necessary or until a threshold number of relocations has been performed. In the latter case, the last element is put in a special stash. A lookup in this scheme is very efficient as it only compares $e$ to the $k$ items in $B_{h_{1}(e)}, \ldots, B_{h_{k}(e)}$ and to the $s$ items in the stash. The size of the hash table depends on the number of hash functions $k$ as well as on the stash size $s$. The higher $k$ is chosen, the more likely it is that the insertion process succeeds and hence the smaller the number of bins $b$ becomes. On the other hand, the higher $s$ is chosen, the more insertion failures can be tolerated.

3.2.1 Cuckoo Hashing for PSI. A major problem occurs when using cuckoo hashing for PSI: every item can be mapped to one of $k$ bins, and therefore it is unclear with which of $P_{1}$ 's bins should $P_{2}$ compare its own input elements. Furthermore, the protocol must hide from each party the choice of bins made by the other party to store an element, since that choice depends on other input elements and might reveal information about them. The solution to this is that $P_{2}$ uses cuckoo hashing whereas $P_{1}$ maps each of its elements using simple hashing with each of the $k$ 
hash functions. In addition, for cuckoo hashing, we must ensure that the hashing succeeds except with probability $<2^{-\eta}$, since a hashing error on the side of $P_{2}$ reveals information about its set or results in an incorrect result. As in PSI with simple hashing (cf. Section 3.1), $P_{1}$ will need to pad its bins to size $\max _{b}$ using dummy elements $d_{1} \neq d_{2}$.

3.2.2 Cuckoo Hashing Parameter Analysis. Cuckoo hashing has three parameters that affect the hashing failure probability: the stash size $s$, the number of hash functions $k$, and the number of bins $b=\epsilon n$ (Kirsch et al. 2009). It was shown in Kirsch et al. (2009) that cuckoo hashing of $n$ elements into $(1+\zeta) n$ bins with $\zeta \in(0,1)$ for any $k \geq 2(1+\zeta) \ln \left(\frac{1}{\zeta}\right)$ and $s \geq 0$ fails with probability $O\left(n^{1-c(s+1)}\right)$, for a constant $c>0$ and $n \mapsto \infty$. The constants in the big "O" notation are unclear, which makes it hard to compute a concrete failure probability given a set of parameters.

In the following, we empirically determine the failure probability given the stash size $s$, the number of hash functions $k$, and the number of bins $b$. We analyze the effect of all three parameters separately. We first fix the number of bins $b=2.4 n$ and hash functions $k=2$ (as was done in Kirsch et al. (2009)) and determine the necessary stash sizes $s$. In order to improve performance, we increase the number of hash functions $k$ and determine the number of bins $b$ for which no stash is required (i.e., $s=0$ ). While both approaches achieve good overhead when $n_{1}=n_{2}$, they perform poorly when the parties have unequal set sizes $n_{1} \gg n_{2}$. Hence, in the last step, we show how to obtain a low value for the stash size $s$ and a low number of hash functions $k$ by increasing the number of bins $b=\epsilon n$, which results in a collection of trade-offs for unequal set size applications.

Adjusting the Stash Size s. In the following, we identify the exact stash size $s$ that ensures that the hashing failure probability is smaller than a given $2^{-\eta}$. To obtain concrete numbers, we ran $2^{30}$ repetitions of cuckoo hashing, where we mapped $n$ items to $b=\epsilon n=2.4 n$ bins, for $n \in\left\{2^{11}, 2^{12}, 2^{13}, 2^{14}\right\}$, using $k=2$ hash functions and recorded the stash size $s$ that was needed for cuckoo hashing to be successful. We fix $\epsilon=2.4$ as was done in the original cuckoo hashing with a stash paper (Kirsch et al. 2009). The solid lines in Figure 1 depict the probability that a stash of size $s$ prevented a hashing failure.

From the results we can observe that, to achieve $2^{-30}$ failure probability of cuckoo hashing, we require a stash of size $s=6$ for $n=2^{11}, s=5$ for $n=2^{12}$, and $s=4$ for both $n=2^{13}$ and $n=2^{14}$ elements. However, in our experiments, we need the stash sizes for smaller as well as larger values of $n$ to achieve a cuckoo hashing failure probability of $2^{-30}$. To obtain the failure probabilities for larger values of $n$, we extrapolate the results using linear regression and illustrate the results as dotted lines in Figure 1. We give the extrapolated stash sizes for achieving a hashing failure probability of $2^{-30}$ and $2^{-40}$ for $n \in\left\{2^{8}, 2^{12}, 2^{16}, 2^{20}, 2^{24}\right\}$ in Table 5 . We observe that the stash size for achieving a failure probability of $2^{-30}$ is drastically reduced for higher values of $n$ : for $n=2^{16}$ we need a stash of size $s=4$, for $n=2^{20}$ we need $s=3$, and for $n=2^{24}$ we need $s=2$. This observation is in line with the asymptotic failure probability of $O\left(n^{-s}\right)$.

Adjusting the Number of Hash Functions $k$. The original cuckoo hashing procedure (Pagh and Rodler 2004) fixed the number of hash functions $k=2$. It was later shown in Dietzfelbinger et al. (2010) that increasing the number of hash functions $k>2$ achieves much better utilization of bins in the hash table, i.e., while the average utilization for $k=2$ hash functions is around $50 \%$, the utilization increases to $91.8 \%$ for $k=3,97.7 \%$ for $k=4$, and $99.2 \%$ for $k=5$. Hence, higher values of $k$ allow us to drastically decrease the number of bins. However, similar to the previous stash allocation, the analysis in Dietzfelbinger et al. (2010) was only asymptotic and does not allow us to compute the concrete hashing failure probability.

To determine the concrete failure probability, we again perform $2^{30}$ iterations of cuckoo hashing on $n=1,024$ elements using $k \in\{2,3,4,5\}$ hash functions. Our goal in this analysis is to determine 


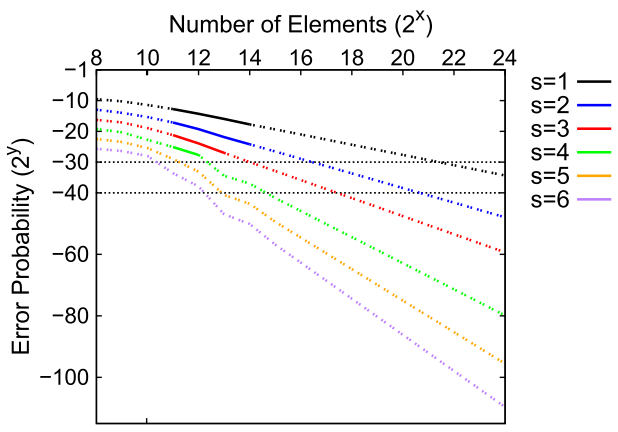

Table 5. Required Stash Sizes $s$ to Achieve $2^{-\eta}$ Failure Probability When Mapping $n$ Elements Into $2.4 n$ Bins

\begin{tabular}{|l|c|c|c|c|c|}
\hline \# Elements $\boldsymbol{n}$ & $2^{8}$ & $2^{12}$ & $2^{\mathbf{1 6}}$ & $2^{20}$ & $2^{24}$ \\
\hline \hline Stash $\boldsymbol{s}(\boldsymbol{\eta}=\mathbf{3 0 )}$ & 8 & 5 & 3 & 2 & 1 \\
\hline Stash $\boldsymbol{s}(\boldsymbol{\eta}=\mathbf{4 0})$ & 12 & 6 & 4 & 3 & 2 \\
\hline
\end{tabular}

Fig. 1. Error probability when mapping $n$ elements to $2.4 n$ bins using cuckoo hashing with $k=2$ hash functions for stash sizes $1 \leq s \leq 6$. The solid lines correspond to actual measurements, the dashed lines were extrapolated using linear regression.

Table 6. Maximum Bin Sizes $\max _{b}$ Required to Ensure No Overflow Occurs When Mapping $n$ Items to $b$ Bins Using $k$ Hash Functions, According to Equation (3)

\begin{tabular}{|l||c|c|c|c|c||c|c|c|c|c|}
\hline \multicolumn{1}{|l||}{ Hash Failure Parameter $\boldsymbol{\eta}$} & \multicolumn{4}{|c||}{30} & \multicolumn{5}{|c|}{40} \\
\hline Set Sizes $\boldsymbol{n}_{\mathbf{1}}=\boldsymbol{n}_{2}$ & $2^{8}$ & $2^{12}$ & $2^{16}$ & $2^{20}$ & $2^{24}$ & $2^{8}$ & $2^{12}$ & $2^{16}$ & $2^{20}$ & $2^{24}$ \\
\hline \hline $\max _{\boldsymbol{b}}$ for $k=\mathbf{2}\left(n=2 n_{1}, b=\{2.4 / 2.4\} n_{2}\right)$ & 13 & 14 & 15 & 16 & 17 & 15 & 16 & 17 & 18 & 19 \\
\hline $\max _{\boldsymbol{b}}$ for $k=\mathbf{3}\left(n=3 n_{1}, b=\{1.20 / 1.27\} n_{2}\right)$ & 19 & 21 & 22 & 23 & 25 & 22 & 23 & 25 & 26 & 27 \\
\hline $\max _{\boldsymbol{b}}$ for $k=\mathbf{4}\left(n=4 n_{1}, b=\{1.07 / 1.09\} n_{2}\right)$ & 23 & 25 & 26 & 28 & 29 & 27 & 28 & 29 & 31 & 32 \\
\hline $\max _{\boldsymbol{b}}$ for $k=\mathbf{5}\left(n=5 n_{1}, b=\{1.04 / 1.05\} n_{2}\right)$ & 26 & 28 & 29 & 31 & 32 & 30 & 31 & 33 & 34 & 36 \\
\hline
\end{tabular}

the minimum number of bins $b_{\min }=\epsilon_{\min } n$ for which the hashing procedure succeeds without a stash except with probability $2^{-30}$. To determine the value of $b_{\min }$, we run cuckoo hashing on an initialization value $\epsilon_{\min }=1.0$ and increase $\epsilon_{\min }$ by 0.01 each time more than one hashing failure has occurred. An interesting observation that we made during the experiments with multiple hash functions was that after a certain threshold value, the hashing failure probability decreased drastically, e.g., only increasing $\epsilon$ by as little as 0.1 when using $k=5$ hash functions could reduce the required stash size from $s=2$ to $s=0$. Overall, we determined the following bin sizes that resulted in a hashing failure probability of $\left\langle 2^{-30}: \epsilon_{\min }=1.20\right.$ for $k=3, \epsilon_{\min }=1.07$ for $k=4$, and $\epsilon_{\min }=1.04$ for $k=5$. We extrapolate the values of $\epsilon_{\min }$ for $\eta=40$ by adding an additional security margin of factor $4 / 3$ to the $\epsilon_{\min }$ values for $\eta=30$ which results in $\epsilon_{\min }=1.27$ for $k=3$, $\epsilon_{\text {min }}=1.09$ for $k=4$, and $\epsilon_{\text {min }}=1.05$ for $k=5$.

A consequence of increasing the number of hash functions is that the party $P_{1}$, who uses simple hashing, needs to increase the maximum bin size $\max _{b}$. This is due to two factors: on one hand $P_{1}$ needs to map each element $k$ times to its hash table. On the other hand, the parties decrease the number of bins due to the reduced $\epsilon$. We re-compute the maximum bin size of $P_{1}$ given the increased number of hash functions using Equation 3 and give the results in Table 6. Given these results, we can compute the total number of comparisons by multiplying the number of bins $b$ with $\max _{b}$. From these results, we observe that $k=3$ achieves the best performance for equal set sizes.

Adjusting the Number of Bins $b$. The required stash sizes for $b=2.4 n$ bins and $k=2$ hash functions are relatively large for small set sizes (e.g., $s=8$ for $n=256$ ). In case of equal set sizes $n_{1}=n_{2}$, 


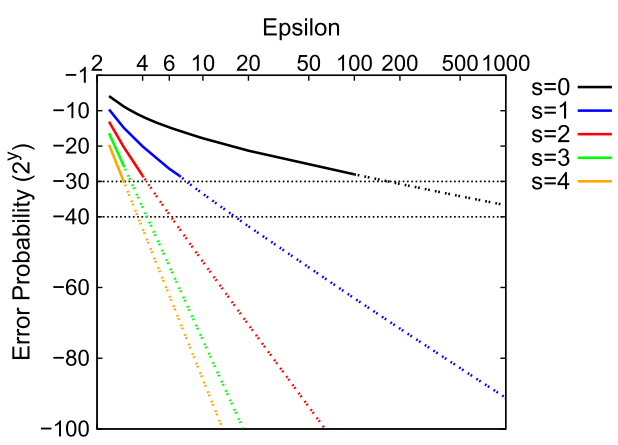

Table 7. Required Number of Bins $b=256 \epsilon$ to Achieve $<2^{-\eta}$ Hashing Failure Probability Given a Fixed Stash Size $s$

\begin{tabular}{|l|c|c|c|c|c|}
\hline Stash Size $\boldsymbol{s}$ & $\mathbf{0}$ & $\mathbf{1}$ & $\mathbf{2}$ & $\mathbf{3}$ & $\mathbf{4}$ \\
\hline \hline $\boldsymbol{\epsilon}(\boldsymbol{\eta}=\mathbf{3 0})$ & 166 & 7.8 & 4.2 & 3.4 & 3 \\
\hline $\boldsymbol{\epsilon}(\boldsymbol{\eta}=\mathbf{4 0})$ & 2,500 & 16 & 6.2 & 4.4 & 3.8 \\
\hline
\end{tabular}

Fig. 2. Error probability when mapping 256 elements to $b=256 \epsilon$ bins using cuckoo hashing with $k=2$ hash functions for stash sizes $0 \leq$ $s \leq 4$. The solid lines correspond to actual measurements, the dashed lines were extrapolated using logarithmic regression.

this does not impact the performance of the protocols much. In the case of unequal set sizes $n_{1} \gg$ $n_{2}$, however, large stash sizes will greatly decrease the performance, since each element in the stash needs to be compared with each item in the large set with possibly millions of elements. Furthermore, even when increasing the number of hash functions $k>2$ to remove the stash, $P_{1}$ would need to map each of its million elements $k$ times into its hash table, which increases $\max _{b}$ and hence incurs a great overhead.

To improve the performance for unequal set sizes, we fix the stash sizes $s \in\{0,1,2,3,4\}$ and the number of hash functions to $k=2$ and try to identify the number of bins $b=\epsilon n$ such that the hashing failure probability is less than $2^{-\eta}$. Similar to the previous experiments, we ran $2^{30}$ repetitions of cuckoo hashing, mapping $n$ items to $b=\epsilon n$ bins, for $n=256$ and $\epsilon=$ $\{2.4,3,4,5,6,7,8,9,10,20,100,200\}$, and recorded the stash size $s$ that was needed for cuckoo hashing to be successful. We chose $n=256$ since it is a good approximation of the number of contacts in a user's addressbook and it is used in our experiments in Section 6.2.3.

The results of our experiments are depicted as solid lines in Figure 2. From the results, we can observe that the probability of requiring a stash size of $s$ decreases logarithmically with growing $\epsilon$ : while for small $\epsilon$ the probabilities decrease quickly, they decrease slower for large $\epsilon$, e.g., when increasing $\epsilon$ from 2.4 to 4 , the hashing failure probability for a stash of size $s=0$ decreases from $2^{-6}$ to $2^{-12}$. If, on the other hand, $\epsilon$ is increased from 20 to 100 , the hashing failure probability for $s=0$ only decreases from $2^{-21}$ to $2^{-28}$. Since we are interested in identifying $\epsilon$ such that the probability of requiring a stash of size $s$ decreases below $2^{-\eta}$, we use regression via a logarithmic function to extrapolate the probabilities. These estimated probabilities are depicted as dotted lines in Figure 2 and the smallest $\epsilon$ for which the hashing failure probability decreases below $2^{-30}$ and $2^{-40}$ is given in Table 7 .

The estimations indicate that, to reduce the stash size to $s=0$, we would need to set $\epsilon=166$ to guarantee $2^{-30}$ hashing failure probability and to $\epsilon=2,500$ to guarantee $2^{-40}$ hashing failure probability. When allowing a bigger stash size $s=1, \epsilon$ decreases drastically, allowing us to set $\epsilon=7.8$ for $2^{-30}$ hashing failure probability and $\epsilon=16$ for $2^{-40}$ hashing failure probability. In our experiments, the exact choice of $\epsilon$ and $s$ depends on the difference between the set sizes $n_{1}$ and $n_{2}$ as well as the protocol that is used (cf. Section 6.2.3), i.e., if $n_{2}$ is only a few hundred while $n_{1}$ is several million, it can be more efficient to choose $\epsilon=166$ to achieve stash size $s=0$. 


\subsection{Permutation-Based Hashing}

The overhead of our circuit-based PSI protocols in Section 4 and of the OT-based PSI protocol in Section 5 depends on the bit-length $\sigma$ of the items that the parties map to bins. The bit-length of the stored items can be reduced based on a permutation-based hashing technique that was suggested in Arbitman et al. (2010) for reducing the memory usage of cuckoo hashing. That construction was presented in an algorithmic setting to improve memory usage. As far as we know, this is the first time that it is used in secure computation or in a cryptographic context.

The construction uses a Feistel-like structure. Let $x=x_{L} \mid x_{R}$ be the bit representation of an input item, where $\left|x_{L}\right|=\log b$, i.e., is equal to the bit-length of an index of an entry in the hash table. (We assume here that the number of bins $b$ in the hash table is a power of 2. It was shown in Arbitman et al. (2010) how to handle the general case.) Let $f()$ be a random function whose range is $[0, b-1]$. Then item $x$ is mapped to bin $x_{L} \oplus f\left(x_{R}\right)$. The value that is stored in the bin is $x_{R}$, which has a length that is shorter by $\log b$ bits than the length of the original item. This is a great improvement, since the length of the stored data is significantly reduced, especially if $|x|$ is not much greater than $\log b$. As for the security, since the function $f$ is random, the maximum load of a bin is $\log n$ with high probability.

The structure of the mapping function ensures that if two items $x, x^{\prime}$ store the same value $x_{R}=$ $x_{R}^{\prime}$ in the same bin, then it must hold that $x=x^{\prime}$ : if the two items are mapped to the same bin, then $x_{L} \oplus f\left(x_{R}\right)=x_{L}^{\prime} \oplus f\left(x_{R}^{\prime}\right)$. If the stored values are equal and hence satisfy $x_{R}=x_{R}^{\prime}$, it must also hold that $x_{L}=x_{L}^{\prime}$, and therefore $x=x^{\prime}$.

As a concrete example, assume that $|x|=32$ and that the table has $b=2^{20}$ bins. Then the values that are stored in each bin are only 12 bits long, instead of 32 bits in the original scheme. Note also that the computation of the bin location requires a single instantiation of $f$, which can be implemented with a medium-size lookup table. Note that, when mapping an element into a bin using multiple hash functions, e.g., when using cuckoo hashing, the index of the hash function needs to be added to the representation in the bin to preserve uniqueness. This observation was also pointed out in Lambæk (2016).

\section{CIRCUIT-BASED PSI}

Unlike special purpose PSI protocols, the protocols that we describe in this section are based on generic secure computation techniques that can be used for computing arbitrary functionalities. Two of the most prominent generic secure computation protocols in the semi-honest model are the Goldreich-Micali-Wigderson (GMW) protocol (Goldreich et al. 1987) and Yao's garbled circuits protocol (Yao 1986). A detailed description and comparison of these two protocols is given in Schneider and Zohner (2013). Both protocols securely evaluate a function by representing it as Boolean circuit, where the parties evaluate cryptographic operations and perform communication for each AND gate in the circuit. Hence, the complexity of generic protocols is often measured in the circuit size, which is the overall number of AND gates. In addition, the complexity of the GMW protocol is also measured in the circuit depth, which is the highest number of AND gates from any input to any output, since the GMW protocols need to perform interaction for each AND gate. In this section, we outline the sort-compare-shuffle (SCS) circuit of Huang et al. (2012), a Boolean circuit of size $O(n \log n)$ for computing the PSI functionality (Section 4.1). We then show how to use the hashing methods described in Section 3 to achieve better complexity than the SCS circuit using a naïve pairwise-comparison circuit (Section 4.2). Finally, we revisit the method of Pinkas et al. (2009) where generic secure computation techniques are used to instantiate an OPRF (cf. Section 2.1), which is used to process the input elements of one party (Section 4.3). 
The usage of generic protocols has the advantage that the functionality of the protocol can easily be extended, without having to change the protocol or the security of the resulting protocol. For example, it is straightforward to change the SCS and PWC protocols to compute the size of the intersection, or a function that outputs if the intersection is greater than some threshold, or compute a summation of values (e.g., revenues) associated with the items that are in the intersection. Computing these variants using other PSI protocols is non-trivial.

\subsection{Sort-Compare-Shuffle Circuit for PSI}

A Boolean circuit for PSI that has $O(n \log n)$ size is the SCS circuit described in Huang et al. (2012). (We refer here to the SCS circuit that uses the Waksman permutation for shuffling.) The SCS circuit computes the intersection between two sets by first sorting both sets into a single sorted list, then comparing all neighboring elements for equality, and finally shuffling the intersecting elements to hide any information that could be obtained from the resulting order.

The overall size of the SCS circuit for inputs of bit-length $\sigma$ is $\sigma\left(3 n \log _{2} n+4 n\right)-n$ AND gates, which is the sum of $2 \sigma n \log _{2}(2 n)$ AND gates for the sort circuit, $\sigma(3 n-1)-n$ AND gates for the compare circuit, and $\sigma\left(n \log _{2} n-n+1\right)$ for the shuffle circuit. It is important to note that approximately $2 / 3$ of the AND gates in the circuit are due to multiplexers. These multiplexer gates can be efficiently evaluated in GMW using vector multiplication triples (Demmler et al. 2015), reducing the cost in GMW from $\sigma$ AND gates to the equivalent of 1 AND gate for a $\sigma$-bit multiplexer.

Instantiation. For our experiments in Section 6, we used GMW to evaluate a depth-optimized variant of the SCS circuit, where the comparison gates have $3 \sigma-\log _{2}(\sigma)-2$ AND gates instead of $\sigma$ but have a depth of $\log _{2} \sigma$ instead of $\sigma$ for $\sigma$-bit values (cf. Schneider and Zohner (2013)). Consequently, the size of the SCS circuit is increased from approximately $3 n \sigma \log _{2} n$ to $5 n \sigma \log _{2} n$, but its depth is decreased from $\sigma \log _{2} n$ to $\log _{2}(n) \log _{2}(\sigma)$. Using the vector multiplication-triple optimization of Demmler et al. (2015), the size of the depth-optimized SCS circuit is again decreased back to approximately $3 n \sigma \log _{2} n$.

\subsection{Pairwise Comparison (PWC) and Hashing}

A simpler circuit for performing the PSI functionality is a pairwise-comparison (PWC) circuit, where each element in the set of $P_{1}$ is compared to each element in the set of $P_{2}$. However, this circuit would scale with $O\left(n_{1} n_{2}\right)$, making it impractical for larger sets. Using the hashing methods from Section 3, we can drastically reduce the number of comparisons as follows:

- Both parties use a table of size $b=\mathrm{O}\left(n_{2}\right)$ to store their elements. Our analysis (Section 3.2.2) shows that setting $b=\epsilon n_{2}$ reduces the error probability to be negligible for reasonable input sizes $\left(2^{8} \leq n_{2} \leq 2^{24}\right)$ when setting the stash size accordingly (cf. Section 3.2).

$-P_{2}$ maps its input elements to $b$ bins using cuckoo hashing with $k$ hash functions and a stash; empty bins are padded with a dummy element $d_{2}$.

$-P_{1}$ maps its input elements into $b$ bins using simple hashing. The size of the bins is set to be $\max _{b}$, a parameter that is set to ensure that no bin overflows (cf. Section 3.1.2). The remaining slots in each bin are padded with a dummy element $d_{1} \neq d_{2}$. The analysis described in Section 3.1.2 shows how $\max _{b}$ is computed and is set to a value smaller than $\log n_{2}$.

- The parties securely evaluate a circuit that compares the element that was mapped to a bin by $P_{2}$ to each of the $\max _{b}$ elements mapped to it by $P_{1}$.

-Finally, each element in $P_{2}$ 's stash is checked for equality with all $n_{1}$ input elements of $P_{1}$ by securely evaluating a circuit computing this functionality. 
- To reduce the bit-length of the elements in the bins, and, respectively, the circuit size, the protocol uses permutation-based hashing as described in Section 3.3. (Note that using this technique is impossible with SCS circuits of Huang et al. (2012).)

Efficiency. Let $m$ be the number of element comparisons that are performed in the circuit with $m=b \cdot \max _{b}+s n_{1}$, i.e., for each of the $b$ bins, the parties perform $\max _{b}$ comparisons per bin as well as $n_{1}$ comparisons for each of the $s$ positions in the stash. Each element is of length $\sigma^{\prime}$ bits, which is the reduced length of the elements after being mapped to bins using permutation-based hashing, i.e., $\sigma^{\prime}=\sigma-\log _{2} b$. A comparison of two $\sigma^{\prime}$-bit elements is done by computing the bitwise XOR of the elements and then a tree of $\sigma-1$ OR gates, with depth $\left\lceil\log _{2} \sigma^{\prime}\right\rceil$. The topmost gate of this tree is a NOR gate. Afterwards, the circuit computes the XOR of the results of all comparisons involving each item of $P_{2}$. (Note that, at most, one of the comparisons results in a match, therefore the circuit can compute the XOR, rather than the OR, of the results of the comparisons.) Overall, the circuit consists of about $m \cdot\left(\sigma^{\prime}-1\right) \approx n_{1} \cdot\left(\max _{b}+s\right) \cdot\left(\sigma^{\prime}-1\right)$ AND gates and has a depth of $\left\lceil\log _{2} \sigma\right\rceil$.

Advantages. The PWC circuit offers several advantages over the SCS circuit:

- Compared to the number of AND gates in the SCS circuit, which is $3 n \sigma \log n$ (cf. Huang et al. (2012), and recalling that $\sigma^{\prime}<\sigma$, and that $\max _{b}$ was shown to be no greater than $2 \log n$ for $k=2$ and $2^{8} \leq n \leq 2^{24}$ in Table 3 (and not greater than $\log n$ asymptotically), the number of non-linear gates in the PWC circuit is smaller by more than a factor 1.5 compared to the number of non-linear gates in the SCS circuit (even though both circuits have the same asymptotic sizes).

- The main advantage of the PWC circuit is the low AND depth of $\log _{2} \sigma$, which is also independent of the number of elements $n$. This affects the overhead of the GMW protocol that requires a round of interaction for every level in the circuit.

- Another advantage of the PWC circuit is its simple structure: The same small comparison circuit is evaluated for each bin. This property allows for a SIMD (Single Instruction Multiple Data) evaluation with a very low memory footprint and easy parallelization.

-Finally, the efficiency of the SCS circuit is tailored for equal set sizes. For unequal set sizes $n_{1} \gg n_{2}$, its circuit size does not scale well and improves by at most a factor of 2 . In contrast, the PWC circuit scales much better for unequal set sizes and in our experiments improves by factor $3.2 \mathrm{x}$ to $5.4 \mathrm{x}$.

\subsection{Secure Evaluation of an OPRF}

Another method for circuit-based PSI was outlined in Freedman et al. (2005) and Pinkas et al. (2009) and uses an OPRF (cf. Section 2.1). In this protocol, the parties use secure computation to evaluate a pseudo-random function $F_{k}(y)=z$, which takes as input a random key $k$ from $P_{1}$ and an element $y$ from $P_{2}$ and returns the output $z$ to $P_{2}$. The use of secure computation guarantees the obliviousness, i.e., that $P_{1}$ learns no information about $y$ or $z$ while $P_{2}$ learns no information about $k$. The PSI functionality can then be achieved by evaluating the OPRF on each element in the set of $P_{2}$ and having $P_{1}$ locally evaluate and send $F_{k}\left(x_{i}\right)$ for all elements $x_{i} \in X . P_{2}$ can then identify the intersection by computing the plaintext intersection between his output of the OPRF with the elements sent by $P_{1}$.

Efficiency. The efficiency of the circuit-based OPRF construction depends mainly on the instantiation of the pseudo-random function $F$. While it is possible to instantiate $F$ with a cipher that is optimized for use in secure computation such as Albrecht et al. (2015), we consider an AESbased instantiation in our efficiency analysis, since the security of AES is better established and 
many of today's CPUs have native instructions for AES (AES-NI). The number of AND gates in the AES circuit is 5,120 and its multiplicative depth is 60 (Boyar and Peralta 2010). In total, we have to perform $n_{2}$ parallel oblivious AES evaluations, resulting in a total of $5,120 n_{2}$ AND gates and a depth of $60 . P_{1}$, on the other hand, can perform a plaintext AES evaluation on his elements and only needs to send $n_{1}$ collision-resistant strings length of $\ell=\lambda+\log \left(n_{1}\right)+\log \left(n_{2}\right)$ bit. Hence, due to the large constants, the OPRF-based approach is less efficient in concrete terms than the SCS or PWC circuits, even though it scales with $O(n)$ while both other circuits scale with $O(n \log n)$. However, if the set sizes of the parties greatly differ, i.e., for $n_{1} \gg n_{2}$, the size of the OPRF-based circuit improves by factor $n_{1} / n_{2}$ and this approach can be more efficient than other circuit-based constructions and even more efficient than all other PSI protocols, since the elements in the much larger set of $P_{1}$ can be processed at very low cost (cf. Section 6.2.3).

\section{PRIVATE SET INTERSECTION VIA OT}

In this section, we describe our new OT-based PSI protocol, of which an earlier version appeared in Pinkas et al. $(2014,2015)$. In contrast to the conference versions, we improve our protocol such that its complexity is now independent of the bit length $\sigma$ for realistic set sizes. The core of our OT-based PSI protocol is an efficient OPRF (cf. Section 2.1) instantiation using recent OT extension techniques, in particular the random OT functionality (Nielsen et al. 2012; Asharov et al. 2013) and the $\left(\begin{array}{c}N \\ 1\end{array}\right)$-OT of Kolesnikov and Kumaresan (2013), which are essentially used to implement an OPRF. Our protocol operates in three steps: the parties hash their elements into hash tables, mask their elements using the $O P R F$, and compute the plaintext intersection of these masked elements to identify the intersecting elements. The hashing step uses the methods from Section 3 for hashing the elements to bins. In the following, we describe the OPRF construction (Section 5) in more detail.

Hashing: In the first step of our OT-based PSI protocol, the parties have mapped their elements into hash tables $T_{1}$ and $T_{2}$, where the elements in the tables have bit-length $\mu=\sigma-\log _{2} b+\log _{2} k$ due to permutation-based mapping (cf. Section 3.3). $P_{1}$ has used simple hashing and hence its hash table $T_{1}$ has two dimensions (in the sense of a two-dimensional array in a programming language), where the first dimension addresses the bins and the second dimension addresses the elements in the bins. $P_{2}$ has used cuckoo hashing and hence its hash table $T_{2}$ has only one dimension, which addresses the bins. Our OT-based PSI protocol then evaluates for each bin $i$ an ideal OPRF functionality $F$ (cf. Section 2.1) where $P_{1}$ inputs a random key $t_{i}$ and $P_{2}$ inputs the $\mu$-bit element $T_{2}[i]$ and obtains random output $M_{2}[i]=F_{i}\left(T_{2}[i]\right)$. The OPRF must ensure that $P_{1}$ learns no information on the input of $P_{2}$ and that $P_{2}$ learns no information except the outputs that correspond to its elements. Since $P_{1}$ knows the secret OPRF key $t_{i}$, it can locally evaluate $F$ on its elements $T_{1}[i][j]$ to obtain $M_{1}[i][j]=O P R F_{t_{i}}\left(T_{1}[i][j]\right)$, for $1 \leq j \leq\left|T_{1}[i]\right|$.

Computing an OPRF: The main observation is that we can instantiate the OPRF functionality using OT. This step can be implemented using random OT, since there is no need for the sender to explicitly set the transmitted values. Furthermore, we can use the $\left(\begin{array}{c}N \\ 1\end{array}\right)$-ROT protocol of Kolesnikov and Kumaresan (2013), where the sender receives a key $t$ and a receiver with an input $x$ receives $F_{t}(x)$. In more detail, for $\mu$-bit inputs we use one 1 -out-of- $2^{\mu}$ random OT on $\ell$-bit strings $\left(\left(\begin{array}{c}2^{\mu} \\ 1\end{array}\right)-\mathrm{ROT}_{\ell}^{1}\right)$, where $P_{1}$ plays the sender who has no inputs and receives as output the random OPRF key $t_{i}$, while $P_{2}$ plays the receiver who inputs $T_{2}[i]$ and obtains $F_{t_{i}}\left(T_{2}[i]\right)$, for $1 \leq i \leq b$. $P_{1}$ can then use the random OPRF key $t_{i}$, which corresponds to the values $q_{i}$ and $s$ in the Kolesnikov and Kumaresan (2013) protocol in Prot. 2, to evaluate the OPRF outputs $M_{1}[i][j]=F_{t_{i}}\left(v_{j}\right)$ on its input values $v_{j}=T_{1}[i][j]$ as $M_{1}[i][j]=H\left(q_{i} \oplus\left(s \wedge c_{v_{j}}\right)\right)$, where $c_{v_{j}}$ is $v_{j}$-th codeword from the error-correcting code $C^{N}$, for $1 \leq i \leq b$ and $1 \leq j \leq\left|T_{1}[i]\right|$. In fact, this observation has also been 
used in parallel to our work by Kolesnikov et al. (2016) to realize an OT-based PSI protocol that is independent of the element bit-length $\sigma$.

PROTOCOL 3 (Our OT-BASEd PSI Protocol).

- Input of $P_{1}: X=\left\{x_{1}, \ldots, x_{n_{1}}\right\}$.

- Input of $P_{2}: Y=\left\{y_{1}, \ldots, y_{n_{2}}\right\}$.

- Common Input: Bit-length of elements $\sigma$; number of bins $b=\epsilon n_{2}$ (cf. Section 3.2.2); $k$ random hash functions $\left\{h_{1}, \ldots, h_{k}\right\}:\{0,1\}^{\sigma} \mapsto[1 \ldots b]$; reduced bit-length of items in the hash table $\mu=\sigma-\log _{2} b+\log _{2} k$ (cf. Section 3.3); symmetric security parameter $\kappa$; statistical security parameter $\lambda$; mask-length $\ell=\lambda+\log _{2}\left(k n_{1}\right)+\log _{2}\left(n_{2}\right) ; N=$ $2^{\mu}$; dummy element $d_{2}$; stash size $s$.

- Oracles and cryptographic primitives: Both parties have access to a $\left(\begin{array}{c}N \\ 1\end{array}\right)-\operatorname{ROT}_{\ell}^{1}$ functionality.

(1) Hashing:

(a) $P_{1}$ maps the elements in its set $X$ into a two-dimensional hash table $T_{1}[][]$ using simple hashing and $k$ hash functions $\left\{h_{1}, \ldots, h_{k}\right\}$. The first dimension has size $b$ and addresses the bin in the table while the second dimension addresses the elements in the bins.

(b) $P_{2}$ maps the elements in its set $Y$ into a one-dimensional hash table $T_{2}$ [ ] and stash $S\left[\right.$ ] using cuckoo hashing and $k$ hash functions $\left\{h_{1}, \ldots, h_{k}\right\}$. The hash table has size $b$ and the stash has size $s . P_{2}$ then fills all empty entries in $T_{2}$ and $S$ with $d_{2}$. Let $\left|T_{1}[i]\right|$ be the number of elements that are stored in the $i$-th bin of the hash table $T_{1}$ and $\mu$ be the bit-length of these elements for $1 \leq i \leq b$.

(2) OPRF evaluation (via OT):

For each bin $1 \leq i \leq b$, the parties perform the following steps:

(a) Let $v_{j}=T_{1}[i][j]$ and $w=T_{2}[i]$ for $1 \leq j \leq\left|T_{1}[i]\right|$.

(b) The parties invoke the OPRF functionality (instantiated using $\left(\begin{array}{c}N \\ 1\end{array}\right)$-ROT $\left.\mathrm{RO}_{\ell}^{1}\right)$, where $P_{1}$ learns a random key $t_{i}$ and $\mathrm{PB}$ inputs its element $w$ and obtains $M_{2}[i]=F_{t_{i}}(w)$.

(c) $P_{1}$ who holds the OPRF key $t_{i}$ locally evaluates $F$ on its inputs $v_{j}$ and obtains $M_{1}[i][j]=F_{t_{i}}\left(v_{j}\right)$.

Stash: For each element in the stash $S$, the parties repeat the same steps where, for the $i$-th stash position, $P_{1}$ evaluates the OPRF on his whole input set $X$ and obtains $n_{1}$ masks $M_{S_{1}}[i]$ while $P_{2}$ evaluates the OPRF on $S[i]$ and obtains one mask $M_{S_{2}}[i]$.

(3) Plaintext Intersection

(a) Let $\bigcup_{1 \leq i \leq b, 1 \leq j \leq\left|T_{1}[i]\right|} M_{1}[i][j]$. $P_{1}$ randomly permutes $V$ and sends it to $P_{2}$.

(b) $P_{2}$ computes the intersection $Z=\left\{T_{2}[i] \mid M_{2}[i] \in V\right\}$.

Stash: The parties perform the same operation to identify whether an element on the stash is in the intersection: $P_{1}$ permutes and sends $M_{S_{1}}[i]$ to $P_{2}$, who adds $S[i]$ to the intersection $Z$ if $M_{S_{2}}[i] \in M_{S_{1}}[i]$.

- Output: $P_{1}$ has no output; $P_{2}$ outputs the intersection $Z=X \cap Y$.

Computing the intersection: After $P_{1}$ has evaluated the OPRF for all bins $i$, it collects the OPRF outputs $M_{1}[i][j]$ for all $j \in\left[1 \ldots\left|T_{1}[i]\right|\right]$ to a set $V$, permutes the order of the elements of $V$ and sends the result. $P_{2}$ identifies whether $T_{2}[i]$ is in the intersection by checking whether $M_{2}[i]$ matches 
any element in $V$. If the element $T_{2}[i]$ matches any element in $T_{1}[i]$, their OPRF outputs will be equal. If $T_{2}[i]$ matches no element in $T_{1}[i]$, their OPRF outputs will differ except with probability $\left|T_{1}[i]\right| \cdot 2^{-\ell}$. The elements in the stash of $P_{2}$ are processed independently in a similar fashion: both parties evaluate the OPRF, $P_{2}$ obtains the output for the elements in its stash, and $P_{1}$ evaluates the OPRF locally on each element of its set and sends the permuted outputs to $P_{2}$, who identifies the intersection.

Efficiency. The main computation and communication overhead comes from the OPRF evaluation. The efficiency of the OPRF depends greatly on the underlying instantiation. We instantiate the OPRF that maps $\mu$-bit inputs to $\ell$-bit outputs using the $\left(\begin{array}{c}2^{\mu} \\ 1\end{array}\right)-\operatorname{ROT}_{\ell}^{1}$ protocol of Kolesnikov and Kumaresan (2013) with the linear BCH code $\left[2^{77}, 512,129\right]$, generated by Morelos-Zaragoza (2006) (cf. Section 2.2.3). Overall, the parties perform $s+b$ OPRF evaluations, which correspond to $\left(\begin{array}{c}2^{\mu} \\ 1\end{array}\right)-\mathrm{ROT}_{\ell}^{s+b}$, where the stash size $s$ and the number of bins $b=\epsilon n_{2}$ are chosen to achieve negligible cuckoo hashing error probability (cf. Section 3.2.2). Regarding the communication, $P_{2}$ sends $512(s+b)$ bits for the $\left(\begin{array}{c}2^{\mu} \\ 1\end{array}\right)$-ROT, while $P_{1}$ sends $k \ell n_{1}$ bits for the permuted OPRF output, where $k$ is the number of hash functions used for cuckoo hashing (cf. Section 3.2.2) and $\ell=\log _{2}\left(k n_{1}\right)+\log _{2}\left(n_{2}\right)+\lambda$. Regarding computation, note that in a naïve $\left(\begin{array}{c}2^{\mu} \\ 1\end{array}\right)$-ROT evaluation the sender $P_{1}$ would need to perform $2^{\mu}$ CRF evaluations, one for each message. However, since $P_{1}$ only needs to obtain the output for actual elements in its bins, it only needs to perform $(k+s) n_{1}$ CRF evaluations, which is independent of $\mu$.

Correctness. In the following, we analyze the correctness of the scheme. We assume that in Step 1 in Prot. 3, $P_{1}$ has used simple hashing to map each element $k$ times into the hash table $T_{1}$ while $P_{2}$ has used cuckoo hashing to map each element once into the hash table $T_{2}$.

If $x=y$, then $P_{1}$ and $P_{2}$ will have the same item in a bin in their hash tables $\left(P_{2}\right.$ has mapped the item to one of $k$ bins while $P_{1}$ has mapped the item to all $k$ bins). For this bin $i, P_{2}$ obtains $M_{x}=F_{t_{i}}(x)$ as output of the OPRF and $P_{1}$ can locally compute $M_{y}=F_{t_{i}}(y)$ with $M_{x}=M_{y}$, and $P_{2}$ successfully identifies equality.

If $x \neq y$, then the probability that $M_{x}=M_{y}$ is $2^{-\ell}$. However, we require that all OPRF outputs $M_{2}$ for elements in the hash table $T_{2}$ of $P_{2}$ are distinct from all outputs $M_{1}$ for elements in the hash table $T_{1}$ of $P_{1}$, which happens with probability $k n_{1} n_{2} 2^{-\ell}$. Thus, to achieve correctness with probability $1-2^{-\lambda}$, we must increase the bit-length of the OTs to $\ell=\lambda+\log _{2}\left(k n_{1}\right)+\log _{2}\left(n_{2}\right)$.

Security. The proof of security follows the common security definitions of secure computation (Goldreich 2004) for semi-honest adversaries. We show that the view of both $P_{1}$ and $P_{2}$ in the PSI protocol can be simulated given only their input and output to the protocol. We assume that the $\left(\begin{array}{c}N \\ 1\end{array}\right)$-ROT protocol implements an ideal $\left(\begin{array}{c}N \\ 1\end{array}\right)$-ROT functionality. Namely, in the analysis we can replace this protocol with a trusted party that receives an input $x$ from the receiver and no input from the sender, and outputs a random key $t$ to the sender and the value $F_{t}(x)$ to the receiver.

The simulation of $P_{1}$ 's view is obvious, since the only information that it learns in the PSI protocol are the random keys $t_{i}$ chosen in the random OT, which are independent of $P_{2}$ 's input. Therefore, $P_{1}$ 's view can be simulated by sending it a list of random values, using them to encrypt its inputs, as is defined in the protocol, and sending the result to $P_{2}$.

$P_{2}$ 's view in the protocol consists of the set $M_{2}$ of encrypted outputs of $F$ which it learns in the $\left(\begin{array}{c}N \\ 1\end{array}\right)$-ROT protocols, and the set of encrypted values $M_{1}$ sent to it (in permuted order) by $P_{1}$. If there are two elements $x \in X$ and $y \in Y$ with $x=y$, then there are corresponding values $m_{x} \in M_{1}, m_{y} \in$ $M_{2}$ for which $m_{x}=m_{y}$. All other values in $M_{1}, M_{2}$ are indistinguishable from random, based on the pseudo-randomness of $F$. Therefore, the view of $P_{2}$ can be simulated given the output of the protocol (i.e., knowledge whether each of its inputs $y$ is in the intersection): The set $M_{2}$ consists 
of random values $m_{1}, \ldots, m_{|Y|}$. For each $y \in X \cap Y$ we add $m_{y}$ to $M_{1}$, and add to $M_{1}$ additional $|X|-|Y|$ random values. This is exactly the distribution of values which $P_{2}$ receives when the $\left(\begin{array}{c}N \\ 1\end{array}\right)$-ROT is implemented by a trusted party.

\section{EVALUATION}

In the following, we evaluate the most promising PSI protocols that were outlined before. We first discuss their implementational features and compare them theoretically (Section 6.1). We then give an empirical performance comparison between the protocols for different settings (Section 6.2). Throughout the evaluation, we divide the PSI protocols into four categories, depending on whether the protocol is based on public-key operations, circuits, OT, or provides limited security, and mark the best result of each category in bold.

\subsection{Theoretical Evaluation}

Before evaluating the empirical performance of the PSI protocols, we discuss implementational features of the protocols such as their suitability for large-scale PSI on sets with several million elements (Section 6.1.1) or the ability of the schemes for parallelization (Section 6.1.2), and give their asymptotic computation and communication complexities (Section 6.1.3).

6.1.1 Suitability for Large-Scale PSI. Although hardly discussed, memory consumption poses a very big problem when implementing cryptographic schemes that operate on large amounts of data. As such, many of the implemented PSI protocols quickly exceeded the main memory, requiring more engineering effort and a more careful implementation to allow for PSI on larger sets. In fact, even computing the plaintext intersection for sets of billions of elements becomes a tedious problem, since at least one set needs to be fully stored at one point during the execution. In this case, one can store the data on disk, which decreases performance greatly when arbitrary lookups are performed.

Limited Security \& Public-Key-Based PSI. The naïv-hashing, server-aided, and public-key-based PSI schemes are very memory efficient, since they operate only on single elements and can be easily pipelined, allowing PSI on millions of elements even on standard PCs.

Circuit-Based PSI. The circuit-based PSI schemes have a very high memory consumption. In our implementations, we evaluate and delete gates if they will not be used anymore to decrease the memory consumption. Yao's garbled circuits has a higher memory consumption than GMW, since $\kappa$-bit keys have to be stored for each wire instead of single bits. A pipelined circuit generation and evaluation, as is done in FastGC (Huang et al. 2011; Henecka and Schneider 2013), would allow us to perform PSI on larger sets. The main memory limitation of our Yao and GMW implementation comes from the circuit having to be fully built and stored in memory. To decrease the memory footprint of the circuit, we build circuits that are evaluated many times in parallel in a SIMD fashion, which evaluates the circuit on multiple values in parallel. This SIMD evaluation especially benefits the PWC (Section 4.2) and OPRF (Section 4.3) circuits, since the same circuit is evaluated on all elements in parallel.

OT-Based PSI. The garbled Bloom filter and random garbled Bloom filter PSI protocols of Dong et al. (2013) and Pinkas et al. (2014) need random access to positions in the Bloom filter to identify the intersecting elements. Hence, the entire Bloom filter needs to be kept in memory or alternatively needs to be outsourced to disk, but this would result in higher runtimes. The garbled Bloom filter holds $1.44 n \kappa$ entries of at least $\lambda$-bit shares, resulting in at least $875 \mathrm{MB}$ for sets of one million elements. The main memory limitation of our OT-based PSI protocol (Section 5) are the hash tables, in particular the cuckoo hash table. While the hash table for simple hashing can 
be easily stored on disk, the cuckoo hash table needs to perform arbitrary lookups when evicting elements. The cuckoo hash table holds $1.2 n$ elements of at most $\ell=\lambda+\log \left(n_{1}\right)+\log \left(n_{2}\right)$-bit length, resulting in $12 \mathrm{MB}$ for sets of one million elements and hence scales much better than the Bloom filter-based protocols.

6.1.2 Parallelizability of Schemes. The experiments we perform in the empirical evaluation only consider execution using a single thread. However, if more computational resources are available, the schemes can be run using multiple threads to improve their performance. Note, however, that the bottleneck for many protocols (i.e., all except the public-key-based protocols) quickly shifts from computation to communication, since symmetric cryptographic operations can be evaluated very efficiently using AES-NI. In the following, we discuss the ability of the schemes to be parallelized.

Limited Security \& Public-Key-Based PSI. The naïve-hashing, server-aided, and public-key-based PSI schemes can easily be parallelized since the elements are processed independently of each other. The main bottleneck for parallelization in all these schemes is the plaintext intersection of hash values that is done at the end of each protocol.

Circuit-Based PSI. The circuit-based PSI protocols parallelize differently depending on the underlying secure computation protocol. The GMW protocol uses OT extension to pre-compute multiplication triples. This step presents the main computational workload and can be parallelized well. However, the circuit evaluation of GMW requires a number of sequential interactions between the parties that is linear in the depth of the circuit and which cannot be parallelized. Yao's garbled circuits, on the other hand, is a constant round protocol. Its ability to parallelize depends on the underlying circuit structure. Circuits that can be split into many sub-circuits that are independent of each other, such as the PWC and OPRF circuits, can be parallelized easily and efficiently while circuits where all gates are connected, such as the SCS circuit, require circuit-dependent methods for parallelization.

OT-Based PSI. For all OT-based PSI protocols, it holds that the underlying OT extension protocol can be parallelized well. The main differences in parallelizability are due to the hashing scheme that is used to map the elements into the corresponding structure. In the garbled Bloom filter-based PSI protocol of Dong et al. (2013), $P_{1}$ has to generate the garbled Bloom filter in advance, and this step does not parallelize well. This is improved on by the random garbled Bloom filter protocol of Pinkas et al. (2014), where the garbled Bloom filter is generated as an output of OT extension and can hence be fully parallelized. In our OT-PSI protocol, the main bottleneck for parallelization is the cuckoo-hashing procedure. However, cuckoo hashing can be pre-processed since no input of the other party is required.

6.1.3 Asymptotic Performance Comparison. We depict the asymptotic computation complexity for the party with the majority of the workload and total communication complexity of the PSI protocols in Table 8. The computation complexity is expressed as the number of symmetric cryptographic primitive evaluations (sym) and the number of asymmetric cryptographic primitive operations (pk). We assume 3 sym per OT (2.5 sym for the Bloom filter-based protocols), 4 sym per AND gate in Yao's protocol, and 6 sym per AND gate in the GMW protocol.

The most crucial observation we make from the asymptotic complexities is that, asymptotically, the performance amongst the schemes with the same type is nearly equal. The naïve hashing and server-aided protocol both require 1 sym operation per element, the public-key-based protocols all require 2 pk operations per element and need to send two ciphertexts and a hash value, the circuit-based protocols all have to perform work linear in the number of AND gates in the circuit, and the Bloom filter-based protocols both have to perform work linear in the size of the Bloom 
Table 8. Asymptotic Complexities for PSI Protocols

\begin{tabular}{|c|c|c|c|}
\hline Type & Protocol & $\begin{array}{l}\text { Computation } \\
{[\# \text { Ops sym/pk] }}\end{array}$ & $\begin{array}{c}\text { Communication } \\
\text { [bit] }\end{array}$ \\
\hline \multirow{2}{*}{ Limited Security } & Naïve Hashing & $m$ sym & $n_{1} \ell$ \\
\hline & Server-Aided (Kamara et al. 2014) & $m$ sym & $t+|X \cap Y|$ \\
\hline \multirow{3}{*}{ Public-Key } & DH FFC (Meadows 1986) & $2 t \mathrm{pk}$ & $t \rho+n_{1} \ell$ \\
\hline & DH ECC (Meadows 1986) & $2 t \mathrm{pk}$ & $t \varphi+n_{1} \ell$ \\
\hline & RSA (Cristofaro and Tsudik 2010) & $2 t \mathrm{pk}$ & $t \rho+n_{1} \ell$ \\
\hline \multirow{6}{*}{ Circuit } & Yao SCS (Huang et al. 2012) & $12 m \sigma \log m+3 m \sigma$ sym & $6 m \kappa \sigma \log m+2 m \kappa \sigma$ \\
\hline & GMW SCS (Huang et al. 2012) & $18 m \sigma \log m$ sym & $6 m(\kappa+2) \sigma \log m$ \\
\hline & Yao PWC (Section 4.2) & $\begin{array}{c}\sigma\left(4 \epsilon n_{2} \max _{b}+4 s n_{1}+\right. \\
\left.3 \epsilon n_{2}\right) \text { sym } \\
\end{array}$ & $\begin{array}{c}2 \epsilon n_{2} \kappa \max _{b} \sigma+3 s n_{1} \kappa \sigma+ \\
2 \epsilon n_{2} \sigma\end{array}$ \\
\hline & \begin{tabular}{|l} 
GMW PWC (Section 4.2) \\
\end{tabular} & $6 \sigma\left(\epsilon n_{2} \max _{b}+s n_{1}\right) \mathrm{sym}$ & $2(2+\kappa) \sigma\left(\epsilon n_{2} \max _{b}+s n_{1}\right)$ \\
\hline & Yao OPRF (Section 4.3) & $21,760 n_{2}+3 \sigma n_{2} \mathrm{sym}$ & $10,880 n_{2} \kappa+2 n_{2} \kappa \sigma+n_{1} \ell$ \\
\hline & GMW OPRF (Section 4.3) & $32,640 n_{2} \mathrm{sym}$ & $10,880 n_{2}(\kappa+2)+n_{1} \ell$ \\
\hline \multirow{3}{*}{ OT } & Bloom Filter (Dong et al. 2013) & $3.6 m \kappa \mathrm{sym}$ & $1.44 m \kappa(\kappa+\lambda)$ \\
\hline & Random Bloom Filter (Pinkas et al. 2014) & $3.6 m \kappa \mathrm{sym}$ & $1.44 m \kappa^{2}+m \ell$ \\
\hline & OT (Section 5) + Hashing (Section 3) & $3 \epsilon n_{2}+(k+s) n_{1}$ sym & $512 \epsilon n_{2}+(k+s) n_{1} \ell$ \\
\hline
\end{tabular}

$\left(\sigma\right.$ : Bit Size of Set Elements; $t=n_{1}+n_{2} ; m=\max \left(n_{1}, n_{2}\right)$; pk: Public-key Operations; sym: Symmetric Cryptographic Operations; $\ell=\lambda+\log n_{1}+\log n_{2} ; \kappa, \rho, \varphi, \lambda$ : Security Parameters as Defined in Section $2.1 ; \epsilon, k, s, \max _{b}$ : Hashing Parameters as Defined in Section 3.1 and Section 3.2). Computation Gives the Number of Operations that Need to be Performed in Sequence.

filter. The main discrepancy can be seen among the OT-based protocols, where the communication complexity of the Bloom filter-based protocols scales quadratically with the symmetric security parameter $\kappa$ while our OT-based PSI protocol scales only linear in the security parameter $\kappa$ (we need 512-bit codewords to achieve relative Hamming distance $\kappa$, cf. Section 2.2.3).

\subsection{Empirical Evaluation}

We empirically evaluate and compare the performance of the presented semi-honest PSI protocols. All protocols are instantiated with a 128-bit security level according to NIST recommendations (cf. Section 2.1). We first describe our benchmarking environment and outline our implementations (Section 6.2.1). We then benchmark the protocols in a LAN and a WAN setting and give their concrete communication (Section 6.2.2). Finally, we evaluate the performance of large-scale PSI (Section 6.2.4).

6.2.1 Benchmarking Environment. We ran our experiments in a LAN and a WAN setting. The LAN setting consists of two PCs (Intel Haswell i7-4770K CPU with 3.5 GHz and 16GB RAM) that are connected via a Gigabit Ethernet. The WAN setting consists of two Amazon EC2 m3.medium instances (Intel Xeon E5-2670 CPU with 2.6GHz and 3.75GB RAM) that are located in North Virginia (U.S. east coast) and Frankfurt (Europe) with an average bandwidth of 98MBit/s and an average round-trip time of $94 \mathrm{~ms}$.

We evaluate the performance of the PSI protocols in two scenarios. In the first scenario, $P_{1}$ and $P_{2}$ hold the same number of input elements $n_{1}, n_{2} \in\left\{2^{8}, 2^{12}, 2^{16}, 2^{20}, 2^{24}\right\}$. In the second scenario, $P_{1}$ has a larger set than $P_{2}$ and we set $n_{1} \in\left\{2^{16}, 2^{20}, 2^{24}\right\}$ and $n_{2} \in\left\{2^{8}, 2^{12}\right\}$. Both parties are not allowed to perform any pre-computation. For the sort-compare-shuffle and PWC circuit-based protocols whose complexity depends on the bit-length of elements $\sigma$, we fix $\sigma=32$ (e.g., for PSI 
on $\mathrm{IPv} 4$ addresses). We use the long-term security parameters as described in Section 2.1. We benchmarked the server-aided PSI protocol of Kamara et al. (2014) by executing the trusted server on one machine and the two clients that wish to compute the intersection on the second machine.

Implementations. The implementation of the blind-RSA-based (Cristofaro and Tsudik 2010) and garbled Bloom filter (Dong et al. 2013) protocols were taken from the authors, but we used a hash-table to compute the last step in the blind-RSA protocol that finds the intersection (the original implementation used pairwise comparisons with quadratic runtime overhead) and the OT extension implementation of Asharov et al. (2013) for the Bloom filter protocol. We use the state-of-the-art Yao's garbled circuits and GMW protocol implementations in the $\mathrm{C}++\mathrm{ABY}$ framework (Demmler et al. 2015), which implements point-and-permute (Malkhi et al. 2004), half-gates (Zahur et al. 2015), free-XOR (Kolesnikov and Schneider 2008), fixed-key garbling (Bellare et al. 2013), and OT extension (Asharov et al. 2013). For Yao's garbled circuits protocol, we evaluated a size-optimized version of the sort-compare-shuffle circuit (comparison circuits of size and depth $\sigma$ ) while for GMW we evaluated a depth-optimized version (comparison circuits of size $3 \sigma$ and depth $\log _{2} \sigma$ ) for $\sigma$-bit input values (Schneider and Zohner 2013). We instantiated the PRP of the server-aided PSI protocol in Kamara et al. (2014) and the CRF in the $\left(\begin{array}{l}2 \\ 1\end{array}\right)$-OT extension with fixed-key AES-128, and instantiated the RO and the CRF in the $\left(\begin{array}{c}N \\ 1\end{array}\right)$-OT extension with SHA-256. We instantiated the CRF in the $\left(\begin{array}{c}N \\ 1\end{array}\right)$-OT using SHA-256 instead of AES, since it needs to process inputs of 512 bit-length and AES only allows to process inputs with 128 bit when using fixed-key AES-128 or 256 bit when using the key schedule of AES-256 (Dessouky et al. 2017). We implemented FFC (finite field cryptography) using the GMP library (v. 5.1.2), ECC using the Miracl library (v. 5.6.1), symmetric cryptographic primitives using OpenSSL (v. 1.0.1e), and used the OT extension implementation of Asharov et al. (2013). We perform all operations in FFC in a subgroup of order $q$, where $|q|=2 \kappa$-bits.

We argue that we provide a fair comparison, since all protocols are implemented in the same programming language $(\mathrm{C} / \mathrm{C}++)$, run on the same hardware, and use the same underlying libraries for cryptographic operations.

For each protocol, we measured the time from starting the program until the client outputs the intersecting elements. All runtimes are averaged over 10 executions.

6.2.2 Empirical Comparison. We evaluate the empirical performance of the PSI protocols in the LAN setting and give the concrete communication of the protocols. While the LAN setting does not necessarily represent a real-world setting for PSI, it allows us to benchmark the protocols in an almost ideal network setting and hence focus on the computation complexity of the protocols. We give a classification for $n=2^{20}$ element sets in Figure 3, and depict the detailed runtime in Table 9 and communication in Table 10. We now compare the performance of the different types of PSI protocols and then compare the PSI protocols of the same type.

Comparison Between Types. From Figure 3, we can observe that PSI protocols of the same type have a similar runtime and communication with the exception of the OT-based PSI protocols. The insecure naïve hashing protocol and server-aided PSI protocol outperform the other PSI protocols by at least an order of magnitude in computation and communication. The public-key-based PSI protocols require only little communication (especially the DH-ECC protocol), but have the highest runtime. The circuit-based PWC protocol has a faster runtime than the public-key-based protocols but requires two orders of magnitude more communication and does not scale well to large sets. Finally, the OT-based PSI protocols differ in performance: the GBF protocol of Dong et al. (2013) has a similar runtime and communication as the circuit-based PWC protocol and our OT-based PSI protocol has a faster runtime than the public-key and circuit-based protocols and require at least an order of magnitude less communication compared to the circuit-based protocols. Among all PSI 
B. Pinkas et al.

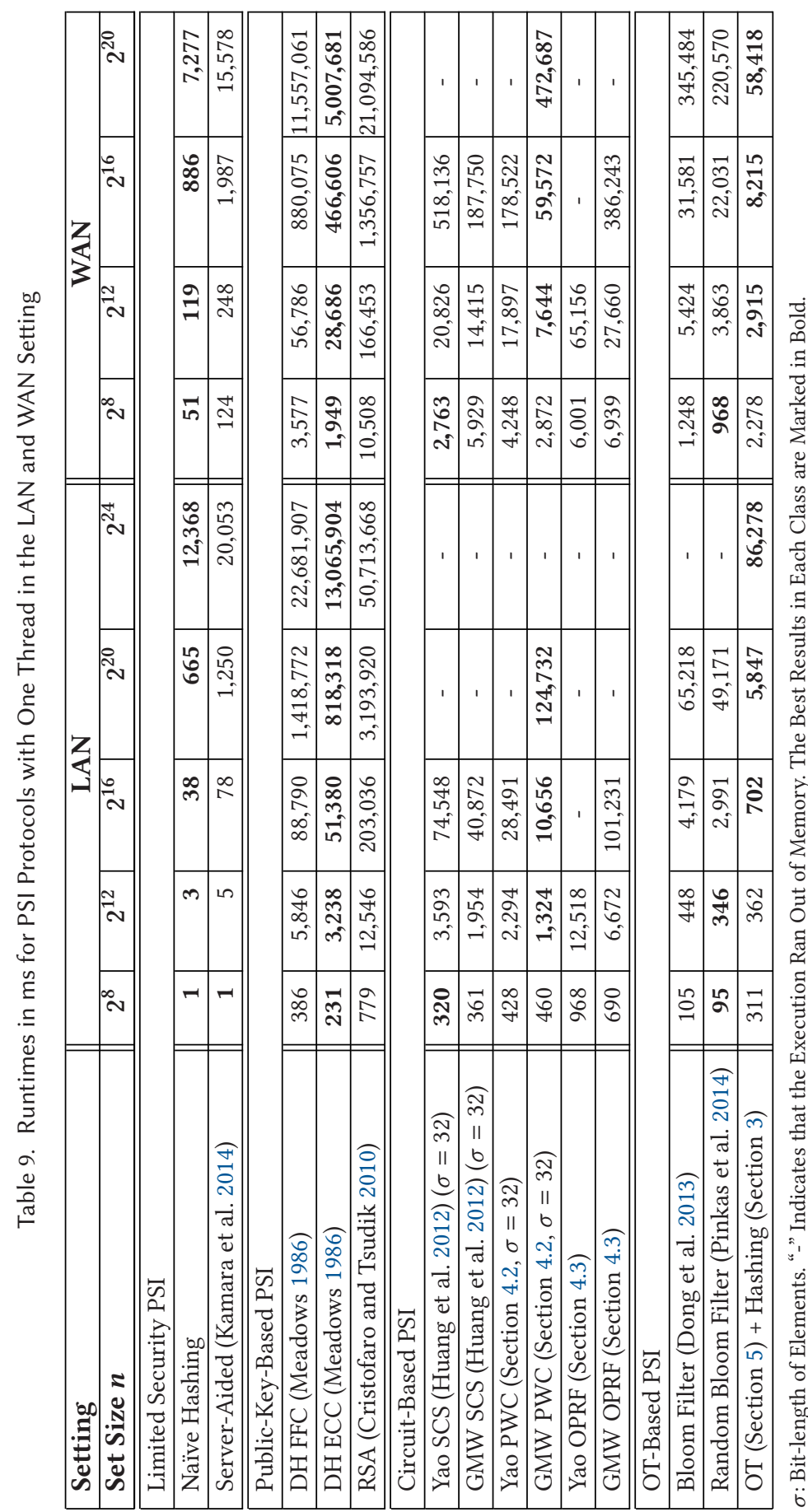




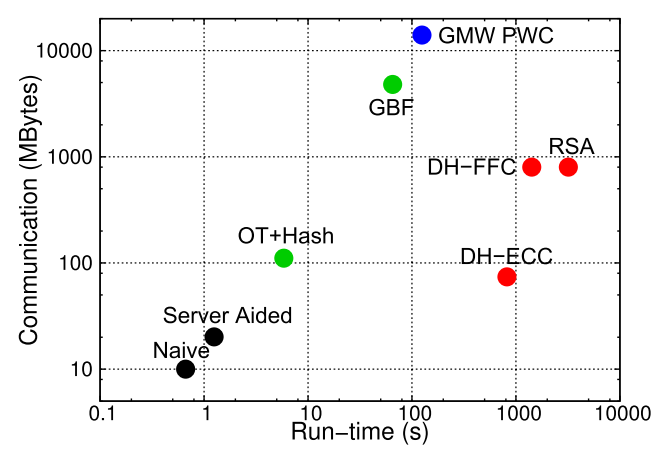

Fig. 3. Runtime in s and communication in MB of PSI protocols for $n=2^{20}$ elements and $\kappa=128$-bit security. Detailed results are given in Table 9 and Table 10.

Table 10. Communication in MB for PSI Protocols

\begin{tabular}{|c|c|c|c|c|c|c|}
\hline Type & Set Size $n$ & $2^{8}$ & $2^{12}$ & $2^{16}$ & $2^{20}$ & $2^{24}$ \\
\hline \multirow{2}{*}{ Limited Security } & Naïve Hashing & 0.002 & 0.031 & 0.600 & 10.000 & 176.000 \\
\hline & Server-Aided (Kamara et al. 2014) & 0.003 & 0.063 & 1.133 & 20.125 & 354.000 \\
\hline \multirow{3}{*}{ Public-Key } & DH-Based FFC (Meadows 1986) & 0.195 & 3.125 & 50.000 & 800.000 & $12,800.000$ \\
\hline & DH-Based ECC (Meadows 1986) & 0.020 & 0.280 & 4.560 & 74.000 & $1,200.000$ \\
\hline & RSA-Based (Cristofaro and Tsudik 2010) & 0.195 & 3.125 & 50.000 & 800.000 & $12,800.000$ \\
\hline \multirow{6}{*}{ Circuit } & Yao SCS (Huang et al. 2012) $(\sigma=32)$ & 7.522 & 168.590 & $3,484.751$ & - & - \\
\hline & GMW SCS (Huang et al. 2012) $(\sigma=32)$ & 7.319 & 162.851 & $3,348.011$ & - & - \\
\hline & Yao PWC (Section 4.2, $\sigma=32$ ) & 8.833 & 124.098 & $1,751.780$ & - & - \\
\hline & GMW PWC (Section 4.2, $\sigma=32$ ) & 5.587 & 78.229 & $1,101.383$ & $14,014.427$ & - \\
\hline & Yao OPRF (Section 4.3) & 44.033 & 704.210 & - & - & - \\
\hline & GMW OPRF (Section 4.3) & 43.193 & 690.890 & $11,054.050$ & - & - \\
\hline \multirow{3}{*}{ OT } & Garbled Bloom Filter (Dong et al. 2013) & 1.037 & 17.314 & 288.560 & $4,801.639$ & - \\
\hline & Random Bloom Filter (Pinkas et al. 2014) & 0.723 & 11.574 & 185.241 & $2,964.855$ & - \\
\hline & OT (Section 5) + Hashing (Section 3) & 0.055 & 0.456 & 6.799 & 111.299 & $1,828.528$ \\
\hline
\end{tabular}

$\sigma$ : Bit-Length of Elements. “-” Indicates the Execution Ran Out of Memory. Best Results in Each Class Marked in Bold.

protocols, our novel OT-based PSI protocol is the fastest and requires about the same amount of communication as public-key-based PSI protocols.

Limited Security-Based PSI. The naïve hashing protocol outperforms the server-aided protocol by a factor of 2 in runtime and communication. However, these protocols have weaker security guarantees than the other protocols that we describe.

Public-Key-Based PSI. For the public-key-based PSI protocols, we observe that the DH-based protocol of Meadows (1986) outperforms the RSA-based protocol of Cristofaro and Tsudik (2010) when using finite field cryptography (FFC). The elliptic curve cryptography (ECC) instantiation of the DH-based protocol becomes even more efficient and outperforms the FFC instantiation by a factor of 2. The advantage of the ECC-based protocol is its communication complexity, which is lowest among all PSI protocols (cf. Table 10). We note that a major advantage of these protocols is their simplicity, which makes them relatively easy to implement.

Circuit-Based PSI. Here we compare the SCS circuit of Huang et al. (2012), our PWC circuit (Section 4.2), and the OPRF circuit (Section 4.3), evaluated using Yao's garbled circuits and GMW. The results can be summarized as follows: 
Table 11. Parameters for Circuit PWC and Our OT-Based PSI Used in the Unequal Set Size Experiments

\begin{tabular}{|c|c|c|c|c|c|c|c|c|c|c|c|c|}
\hline Server Set Size $n_{1}$ & \multicolumn{4}{|c|}{$2^{16}$} & \multicolumn{4}{|c|}{$2^{20}$} & \multicolumn{4}{|c|}{$2^{24}$} \\
\hline \multicolumn{13}{|l|}{ Client Set Size $n_{2}=2^{8}$} \\
\hline Parameter & $k$ & $\epsilon$ & $s$ & $\max _{b}$ & $k$ & $\epsilon$ & $s$ & $\max _{b}$ & $k$ & $\epsilon$ & $s$ & $\max _{b}$ \\
\hline Circuit PWC (Section 4.2, $\sigma=32$ ) & 3 & 1.27 & 0 & 804 & 3 & 1.27 & 0 & 10,426 & 2 & 16 & 1 & 8,938 \\
\hline OT (Section 5) + Hashing (Section 3) & 3 & 1.27 & 0 & 0 & 3 & 1.27 & 0 & 0 & 2 & 2500 & 0 & 0 \\
\hline \multicolumn{13}{|l|}{ Client Set Size $n_{2}=2^{12}$} \\
\hline Parameter & $k$ & $\epsilon$ & $s$ & $\max _{b}$ & $k$ & $\epsilon$ & $s$ & $\max _{b}$ & $k$ & $\epsilon$ & $s$ & $\max _{b}$ \\
\hline Circuit PWC (Section 4.2, $\sigma=32$ ) & 3 & 1.27 & 0 & 98 & 3 & 1.27 & 0 & 816 & 3 & 1.27 & 0 & 10,488 \\
\hline OT (Section 5) + Hashing (Section 3) & 3 & 1.27 & 0 & 0 & 3 & 1.27 & 0 & 0 & 3 & 1.27 & 0 & 0 \\
\hline
\end{tabular}

The GMW protocol is around factor 2 faster than Yao's garbled circuits protocol, which is due to the balanced communication. The PWC circuit scales better than the SCS and OPRF circuits with increasing set sizes and is at least three times more efficient for sets of $2^{16}$ elements. Due to its simple functionality, the PWC circuit can scale up to much larger set sizes and can even process two sets of $2^{20}$ elements sets using GMW.

OT-Based PSI. The random garbled Bloom filter protocol of Pinkas et al. (2014) improves the optimized garbled Bloom filter variant outlined in Dong et al. (2013) by around factor 1.5x in runtime and communication.

Our OT-based PSI protocol has a higher runtime than both Bloom filter-based protocols for small set sizes since the number of base-OTs (and hence public-key operations) that are required for the $\left(\begin{array}{c}N \\ 1\end{array}\right)$-OT extension is four times higher. However, this workload is linear in the security parameter and amortizes with increasing set sizes. For larger set sizes of $n>2^{12}$, our OT-based PSI protocol is up to nine times more efficient in terms of runtime than the random garbled Bloom filter protocol and has between factor $12 \mathrm{x}$ and $25 \mathrm{x}$ less communication.

6.2.3 PSI with Unequal Set Sizes. In many PSI applications, the parties have unequal set sizesoften a client with a set of a few hundred elements performs PSI with a server that has a database of millions of records. We perform PSI with unequal set sizes $n_{1} \in\left\{2^{16}, 2^{20}, 2^{24}\right\}$ and $n_{2} \in\left\{2^{8}, 2^{16}\right\}$ using the previously best performing protocols of each category: naïve hashing, the server-aided protocol of Kamara et al. (2014), the DH-ECC protocol of Meadows (1986), the PWC and OPRF circuits in Section 4.2 and Section 4.3, and our OT-based PSI protocol in Section 5. We evaluate their performance in the LAN and WAN setting, and give the resulting runtimes in Table 12 and concrete communication in Table 13. For the circuit PWC protocol and our OT-based PSI protocol, which both use hashing techniques, we used the parameters given in Table 11. Note that in the naïve hashing protocol, $P_{1}$ with the large set sends its hashes to $P_{2}$ with the small set. One could drastically reduce communication by having $P_{2}$ send its hashes to $P_{1}$ instead. However, we decided to benchmark the protocols in a consistent setting where $P_{2}$ obtains the outputs and the existing two-party PSI protocols all seem to have a communication lower-bound linear in the set sizes of both parties.

The results are similar to the equal set size experiments with one notable exception: the OPRF circuit performs extremely well and achieves a similar runtime as the server-aided protocol and even outperforms naïve hashing for $n_{2}=2^{8}$ and $n_{1}=2^{24}$. This good performance of the OPRF circuit can be explained by the asymmetric costs for processing the sets of the client and server. While each element in the set of the client is encrypted by securely evaluating an AES circuit using generic secure computation techniques, the server only needs to encrypt each element in 


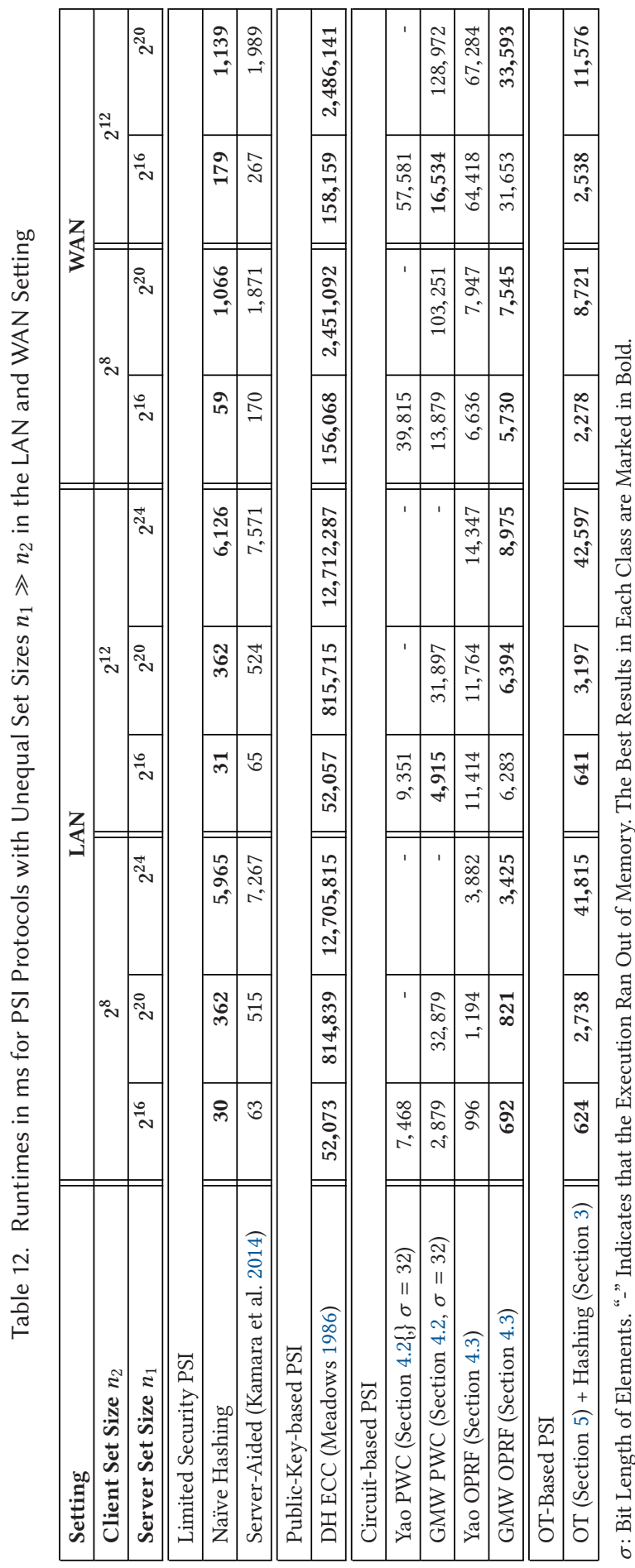


Table 13. Communication in MB for PSI with Unequal Set Sizes $n_{1} \gg n_{2}$

\begin{tabular}{|c|c|c|c|c|c|c|c|}
\hline \multirow{2}{*}{ Type } & \multirow{2}{*}{$\begin{array}{l}\text { Client Set Size } n_{2} \\
\text { Server Set Size } n_{1} \\
\end{array}$} & \multicolumn{3}{|c|}{$2^{8}$} & \multicolumn{3}{|c|}{$2^{12}$} \\
\hline & & $2^{16}$ & $2^{20}$ & $2^{24}$ & $2^{16}$ & $2^{20}$ & $2^{24}$ \\
\hline \multirow{2}{*}{$\begin{array}{l}\text { Limited } \\
\text { Security } \\
\end{array}$} & Naïve Hashing & 0.500 & 9.000 & 144.000 & 0.563 & 9.000 & 160.000 \\
\hline & Server-aided (Kamara et al. 2014) & 0.502 & 9.002 & 144.002 & 0.598 & 9.040 & 160.040 \\
\hline Public-Key & DH ECC (Meadows 1986) & 2.329 & 30.017 & 592.008 & 2.582 & 37.545 & 592.270 \\
\hline \multirow{4}{*}{ Circuit } & Yao PWC (Section 4.2, $\sigma=32$ ) & 336.315 & - & - & 535.082 & - & - \\
\hline & GMW PWC (Section 4.2, $\sigma=32$ ) & 204.18 & $2,643.424$ & - & 333.452 & $2,778.348$ & - \\
\hline & Yao OPRF (Section 4.3) & 40.965 & 49.402 & 184.402 & 646.674 & 655.112 & 790.112 \\
\hline & GMW OPRF (Section 4.3) & 41.454 & 49.891 & 187.441 & 654.564 & 663.001 & 798.001 \\
\hline OT & $\begin{array}{l}\text { OT (Section 5) + Hashing } \\
\text { (Section 3) }\end{array}$ & 1.549 & 27.050 & 432.049 & 2.018 & 27.331 & 480.331 \\
\hline
\end{tabular}

$\sigma$ : Bit-length of Elements. "-” Indicates that the Execution Ran Out of Memory. Best Results Per Class Marked in Bold.

his set using AES with a fixed key and send the resulting ciphertext to the client. Since the set size of the client is small, the overhead for the generic secure computation techniques does not impact the overall runtime significantly. In contrast, the naïve hashing protocol uses SHA-256 to process the elements, which is slower than AES.

6.2.4 PSI on Billion Element Sets. Finally, we demonstrate the scalability of our OT-based PSI protocol by evaluating it on sets of a billion $\sigma=128$-bit elements each. For these sizes, the input elements require $16 \mathrm{~GB}$ of storage, which exceeds the main memory of our servers. Instead, the servers store the elements and intermediate values on their solid state drive (SSD). We also benchmark the naïve hashing protocol as a baseline for performance. We refrained from adding more main memory to process these sets, even though it is the most simple solution, since we are interested in the performance of the protocols if data needs to be stored on the SSD.

To compute the intersection between 2-billion-element sets, naïve hashing requires 74 minutes, of which 19 minutes (26\%) are for hashing and transferring data and 55 minute (74\%) are for computing the plaintext intersection. Our OT-based PSI protocol takes 34.2 hours in total, of which 30.0 hours (88\%) are for simple hashing (cuckoo hashing runs in parallel and requires 16.3 hours), 3 hours (9\%) are for computing the OTs, and 1.2 hours (4\%) are for computing the plaintext intersection.

\section{REFERENCES}

A. Abadi, S. Terzis, and C. Dong. 2015. O-PSI: Delegated private set intersection on outsourced datasets. In ICT Systems Security and Privacy Protection (SEC'15) (IFIP AICT), Vol. 455. Springer, 3-17.

A. Abadi, S. Terzis, and C. Dong. 2017. VD-PSI: Verifiable delegated private set intersection on outsourced private datasets. In Financial Cryptography and Data Security (FC'16)(LNCS), Vol. 9603. Springer, 149-168.

M. R. Albrecht, C. Rechberger, T. Schneider, T. Tiessen, and M. Zohner. 2015. Ciphers for MPC and FHE. In Advances in Cryptology-EUROCRYPT'15 (LNCS), Vol. 9056. Springer, 430-454.

Y. Arbitman, M. Naor, and G. Segev. 2010. Backyard cuckoo hashing: Constant worst-case operations with a succinct representation. In Foundations of Computer Science (FOCS'10). IEEE, 787-796.

G. Asharov, Y. Lindell, T. Schneider, and M. Zohner. 2013. More efficient oblivious transfer and extensions for faster secure computation. In Computer and Communications Security (CCS'13). ACM, 535-548.

G. Asharov, Y. Lindell, T. Schneider, and M. Zohner. 2015. More efficient oblivious transfer extensions with security for malicious adversaries. In Advances in Cryptology-EUROCRYPT'15 (LNCS), Vol. 9056. Springer, 673-701.

N. Asokan, A. Dmitrienko, M. Nagy, E. Reshetova, A.-R. Sadeghi, T. Schneider, and S. Stelle. 2013. CrowdShare: Secure mobile resource sharing. In Applied Cryptography and Network Security (ACNS’13) (LNCS), Vol. 7954. Springer, 432-440. 
P. Baldi, R. Baronio, E. De Cristofaro, P. Gasti, and G. Tsudik. 2011. Countering GATTACA: Efficient and secure testing of fully-sequenced human genomes. In Computer and Communications Security (CCS'11). ACM, 691-702.

R. W. Baldwin and W. C. Gramlich. 1985. Cryptographic protocol for trustable matchmaking. In Symposium on Security and Privacy (S\&P'85). IEEE, 92-100.

D. Beaver. 1996. Correlated pseudorandomness and the complexity of private computations. In Symposium on Theory of Computing (STOC'96). ACM, 479-488.

M. Bellare, V. Hoang, S. Keelveedhi, and P. Rogaway. 2013. Efficient garbling from a fixed-key blockcipher. In Symposium on Security and Privacy (S\&P'13). IEEE, 478-492.

M. Bellare and P. Rogaway. 1993. Random oracles are practical: A paradigm for designing efficient protocols. In Computer and Communications Security (CCS'93). ACM, 62-73.

J. Boyar and R. Peralta. 2010. A new combinational logic minimization technique with applications to cryptology. In Symposium on Experimental Algorithms (SEA'10) (LNCS), Vol. 6049. Springer, 178-189.

E. Bursztein, M. Hamburg, J. Lagarenne, and D. Boneh. 2011. OpenConflict: Preventing real time map hacks in online games. In Symposium on Security and Privacy (S\&P'11). IEEE, 506-520.

H. Carter, C. Amrutkar, I. Dacosta, and P. Traynor. 2013. For your phone only: Custom protocols for efficient secure function evaluation on mobile devices. F. Secur. Commun. Netw. (2013).

E. De Cristofaro, J. Kim, and G. Tsudik. 2010. Linear-complexity private set intersection protocols secure in malicious model. In Advances in Cryptology-ASIACRYPT'10 (LNCS), Vol. 6477. Springer, 213-231.

E. De Cristofaro and G. Tsudik. 2010. Practical private set intersection protocols with linear complexity. In Financial Cryptography and Data Security (FC'10) (LNCS), Vol. 6052. Springer, 143-159.

S. K. Debnath and R. Dutta. 2015. Secure and efficient private set intersection cardinality using bloom filter. In Information Security Conference (ISC’15) (LNCS), Vol. 9290. Springer, 209-226.

D. Demmler, T. Schneider, and M. Zohner. 2015. ABY: A framework for efficient mixed-protocol secure two-party computation. In Network and Distributed System Security (NDSS'15). The Internet Society.

G. Dessouky, F. Koushanfar, A.-R. Sadeghi, T. Schneider, S. Zeitouni, and M. Zohner. 2017. Pushing the communication barrier in secure computation using lookup tables. In Network and Distributed System Security (NDSS'17). The Internet Society.

M. Dietzfelbinger, A. Goerdt, M. Mitzenmacher, A. Montanari, R. Pagh, and M. Rink. 2010. Tight thresholds for cuckoo hashing via XORSAT. In International Colloquium on Automata, Languages and Programming (ICALP'10) (LNCS), Vol. 6198. Springer, 213-225.

C. Dong, L. Chen, and Z. Wen. 2013. When private set intersection meets big data: An efficient and scalable protocol. In Computer and Communications Security (CCS'13). ACM, 789-800.

M. Fischlin, B. Pinkas, A.-R. Sadeghi, T. Schneider, and I. Visconti. 2011. Secure set intersection with untrusted hardware tokens. In Cryptographers' Track at the RSA Conference (CT-RSA'11) (LNCS), Vol. 6558. Springer, 1-16.

M. J. Freedman, C. Hazay, K. Nissim, and B. Pinkas. 2016. Efficient set intersection with simulation-based security. F. Cryptol. 29, 1 (2016), 115-155.

M. J. Freedman, Y. Ishai, B. Pinkas, and O. Reingold. 2005. Keyword search and oblivious pseudorandom functions. In Theory of Cryptography Conference (TCC'05) (LNCS), Vol. 3378. Springer, 303-324.

M. J. Freedman, K. Nissim, and B. Pinkas. 2004. Efficient private matching and set intersection. In Advances in CryptologyEUROCRYPT'04 (LNCS), Vol. 3027. Springer, 1-19.

O. Goldreich. 2004. Foundations of Cryptography. Vol. 2: Basic Applications. Cambridge University Press, Cambridge, UK.

O. Goldreich, S. Micali, and A. Wigderson. 1987. How to play any mental game or a completeness theorem for protocols with honest majority. In Symposium on Theory of Computing (STOC'87). ACM, 218-229.

G. Gonnet. 1981. Expected length of the longest probe sequence in hash code searching. J. ACM 28, 2 (1981), $289-304$.

C. Hazay and Y. Lindell. 2008. Constructions of truly practical secure protocols using standard smartcards. In Computer and Communications Security (CCS'08). ACM, 491-500.

W. Henecka and T. Schneider. 2013. Faster secure two-party computation with less memory. In Symposium on Information, Computer and Communications Security (ASIACCS'13). ACM, 437-446.

Y. Huang, P. Chapman, and D. Evans. 2011. Privacy-preserving applications on smartphones. In Hot topics in Security (HotSec'11). USENIX.

Y. Huang, D. Evans, and J. Katz. 2012. Private set intersection: Are garbled circuits better than custom protocols? In Network and Distributed System Security (NDSS'12). The Internet Society.

Y. Huang, D. Evans, J. Katz, and L. Malka. 2011. Faster secure two-party computation using garbled circuits. In USENIX Security Symposium 2011. USENIX, 539-554.

B. A. Huberman, M. Franklin, and T. Hogg. 1999. Enhancing privacy and trust in electronic communities. In ACM Conference on Electronic Commerce (EC'99). ACM, 78-86. 
Y. Ishai, J. Kilian, K. Nissim, and E. Petrank. 2003. Extending oblivious transfers efficiently. In Advances in CryptologyCRYPTO'03 (LNCS), Vol. 2729. Springer, 145-161.

S. Kamara, P. Mohassel, M. Raykova, and S. Sadeghian. 2014. Scaling private set intersection to s-element sets. In Financial Cryptography and Data Security (FC'14) (LNCS), Vol. 8437. Springer, 195-215.

A. Kirsch, M. Mitzenmacher, and U. Wieder. 2009. More robust hashing: Cuckoo hashing with a stash. SIAM f. Comput. 39, 4 (2009), 1543-1561.

Á. Kiss, J. Liu, T. Schneider, N. Asokan, and B. Pinkas. 2017. Private set intersection for unequal set sizes with mobile applications. Privacy Enhancing Technologies (PoPETs'17) 2017, 4 (2017), 97-117.

L. Kissner and D. Song. 2005. Privacy-preserving set operations. In Advances in Cryptology-CRYPTO'05 (LNCS), Vol. 3621. Springer, 241-257.

V. Kolesnikov and R. Kumaresan. 2013. Improved OT extension for transferring short secrets. In Advances in CryptologyCRYPTO'13 (2) (LNCS), Vol. 8043. Springer, 54-70.

V. Kolesnikov, R. Kumaresan, M. Rosulek, and N. Trieu. 2016. Efficient batched oblivious PRF with applications to private set intersection. In Computer and Communications Security (CCS'16). ACM, 818-829.

V. Kolesnikov and T. Schneider. 2008. Improved garbled circuit: Free XOR gates and applications. In International Colloquium on Automata, Languages and Programming (ICALP'08) (LNCS), Vol. 5126. Springer, 486-498.

M. Lambæk. 2016. Breaking and Fixing Private Set Intersection Protocols. Cryptology ePrint Archive, Report 2016/665. (2016). http://ia.cr/2016/665.

D. Malkhi, N. Nisan, B. Pinkas, and Y. Sella. 2004. Fairplay-A secure two-party computation system. In USENIX Security Symposium 2004. USENIX, 287-302.

C. Meadows. 1986. A more efficient cryptographic matchmaking protocol for use in the absence of a continuously available third party. In Symposium on Security and Privacy (S\&P'86). IEEE, 134-137.

G. Mezzour, A. Perrig, V. D. Gligor, and P. Papadimitratos. 2009. Privacy-preserving relationship path discovery in social networks. In Cryptology and Network Security (CANS'09) (LNCS), Vol. 5888. Springer, 189-208.

M. D. Mitzenmacher. 2001. The power of two choices in randomized load balancing. IEEE Trans. Parallel Distrib. Syst. 12, 10 (2001), 1094-1104.

Robert H. Morelos-Zaragoza. 2006. The Art of Error Correcting Coding. Wiley, Hoboken (New Jersey), US. Code generation tools: http://eccpage.com.

R. Motwani and P. Raghavan. 1995. Randomized Algorithms. Cambridge University Press, New York, NY.

S. Nagaraja, P. Mittal, C.-Y. Hong, M. Caesar, and N. Borisov. 2010. BotGrep: Finding P2P bots with structured graph analysis. In USENIX Security Symposium 2010. USENIX, 95-110.

M. Nagy, E. De Cristofaro, A. Dmitrienko, N. Asokan, and A.-R. Sadeghi. 2013. Do I know you? - Efficient and privacypreserving common friend-finder protocols and applications. In Annual Computer Security Applications Conference (ACSAC'13). ACM, 159-168.

M. Naor and B. Pinkas. 2001. Efficient oblivious transfer protocols. In SIAM Symposium On Discrete Algorithms (SODA'01). Society for Industrial and Applied Mathematics (SIAM'01), 448-457.

A. Narayanan, N. Thiagarajan, M. Lakhani, M. Hamburg, and D. Boneh. 2011. Location privacy via private proximity testing. In Network and Distributed System Security (NDSS'11). The Internet Society.

J. B. Nielsen, P. S. Nordholt, C. Orlandi, and S. S. Burra. 2012. A new approach to practical active-secure two-party computation. In Advances in Cryptology - CRYPTO'12 (LNCS), Vol. 7417. Springer, 681-700.

NIST. 2012. NIST Special Publication 800-57, Recommendation for Key Management Part 1: General (Rev. 3). Technical Report. National Institute of Standards and Technology (NIST), Gaithersburg (Maryland), US.

O. Oksuz, I. Leontiadis, S. Chen, A. Russell, Q. Tang, and B. Wang. 2017. SEVDSI: Secure, Efficient and Verifiable Data Set Intersection. Cryptology ePrint Archive, Report 2017/215. (2017). http://ia.cr/2017/215.

M. Orrù, E. Orsini, and P. Scholl. 2017. Actively secure 1-out-of-N OT extension with application to private set intersection. In Topics in Cryptology-CT-RSA'17 (LNCS), Vol. 10159. Springer, 381-396.

R. Pagh and F. F. Rodler. 2001. Cuckoo hashing. In European Symposium on Algorithms (ESA'01) (LNCS), Vol. 2161. Springer, 121-133.

R. Pagh and F. F. Rodler. 2004. Cuckoo hashing. F. Algorithms 51, 2 (2004), 122-144.

B. Pinkas, T. Schneider, G. Segev, and M. Zohner. 2015. Phasing: Private set intersection using permutation-based hashing. In USENIX Security Symposium 2015. USENIX, 515-530. http://eprint.iacr.org/2015/634.

B. Pinkas, T. Schneider, N. P. Smart, and S. C. Williams. 2009. Secure two-party computation is practical. In Advances in Cryptology-ASIACRYPT'09 (LNCS), Vol. 5912. Springer, 250-267.

B. Pinkas, T. Schneider, and M. Zohner. 2014. Faster private set intersection based on OT extension. In USENIX Security Symposium 2014. USENIX, 797-812. http://eprint.iacr.org/2014/447.

M. Raab and A. Steger. 1998. "Balls into bins"-A simple and tight analysis. In Randomization and Approximation Techniques in Computer Science (RANDOM'98) (LNCS), Vol. 1518. Springer, 159-170. 
P. Rindal and M. Rosulek. 2016. Faster malicious 2-party secure computation with online/offline dual execution. In USENIX Security Symposium 2016. USENIX.

P. Rindal and M. Rosulek. 2017a. Improved private set intersection against malicious adversaries. In Advances in Cryptology-EUROCRYPT'17 (LNCS), Vol. 10210. Springer, 235-259.

P. Rindal and M. Rosulek. 2017b. Malicious-secure private set intersection via dual execution. Forthcoming. In Computer and Communications Security (CCS'17). ACM.

T. Schneider and M. Zohner. 2013. GMW vs. Yao? Efficient secure two-party computation with low depth circuits. In Financial Cryptography and Data Security (FC'13) (LNCS), Vol. 7859. Springer, 275-292.

R. Schürer and W. Schmid. 2006. Monte Carlo and Quasi-Monte Carlo Methods 2004. Springer, Chap. MinT: A Database for Optimal Net Parameters, 457-469.

A. Shamir. 1980. On the power of commutativity in cryptography. In International Colloquium on Automata, Languages and Programming (ICALP'80) (LNCS), Vol. 85. Springer, 582-595.

S. Tamrakar, J. Liu, A. Paverd, J.-E. Ekberg, B. Pinkas, and N. Asokan. 2017. The circle game: Scalable private membership test using trusted hardware. In Computer and Communications Security (ASIACCS'17). ACM, 31-44.

A. C. Yao. 1986. How to generate and exchange secrets. In Foundations of Computer Science (FOCS'86). IEEE, 162-167.

S. Zahur, M. Rosulek, and D. Evans. 2015. Two halves make a whole: Reducing data transfer in garbled circuits using half gates. In Advances in Cryptology-EUROCRYPT'15 (LNCS), Vol. 9057. Springer, 220-250.

Received September 2016; revised September 2017; accepted October 2017 